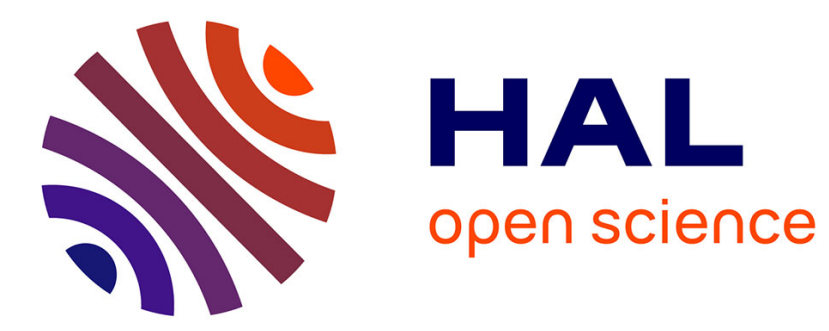

\title{
Benchmark solution for a three-dimensional mixed-convection flow, part 2: analysis of Richardson extrapolation in the presence of a singularity
}

\author{
Xavier Nicolas, S. Gounand, M. Médale, S. Glockner
}

\section{To cite this version:}

Xavier Nicolas, S. Gounand, M. Médale, S. Glockner. Benchmark solution for a three-dimensional mixed-convection flow, part 2: analysis of Richardson extrapolation in the presence of a singularity. Numerical Heat Transfer, Part B Fundamentals, 2011, 60 (5), pp.346-369. hal-00692094

\section{HAL Id: hal-00692094 https://hal.science/hal-00692094}

Submitted on 27 Apr 2012

HAL is a multi-disciplinary open access archive for the deposit and dissemination of scientific research documents, whether they are published or not. The documents may come from teaching and research institutions in France or abroad, or from public or private research centers.
L'archive ouverte pluridisciplinaire HAL, est destinée au dépôt et à la diffusion de documents scientifiques de niveau recherche, publiés ou non, émanant des établissements d'enseignement et de recherche français ou étrangers, des laboratoires publics ou privés. 


\title{
Benchmark solution for a three-dimensional mixed convection flow - Part 2: analysis of Richardson extrapolation in the presence of a singularity
}

\author{
Xavier NICOLAS ${ }^{1}$, Stéphane GOUNAND², Marc MEDALE ${ }^{3}$, \\ Stéphane GLOCKNER ${ }^{4}$
}

${ }^{1}$ Université Paris-Est, Modélisation et Simulation Multi Echelle,
MSME UMR 8208 CNRS, 5 bd Descartes, 77454 Marne-la-Vallée Cedex 2, France (xavier.nicolas@univ-paris-est.fr)

${ }^{2}$ CEA-Saclay, DEN, DM2S, SFME, LTMF, F-91191 Gif-sur-Yvette, France (stephane.gounand@cea.fr)

${ }^{3}$ IUSTI, UMR 6595 CNRS, 5 rue Enrico Fermi, Technopôle de Château-Gombert, 13453 Marseille Cedex 13, France (marc.medale@polytech.univ-mrs.fr)

${ }^{4}$ Université de Bordeaux, IPB ENSCBP, CNRS UMR 5295, Institut I2M, 16 av. Pey-Berland, 33607 Pessac Cedex, France (glockner@enscbp.fr)

Mailing address of the corresponding author:

Xavier NICOLAS, Université Paris-Est Marne la Vallée, Laboratoire MSME UMR 8208 CNRS, Bâtiment Lavoisier, 5 bd Descartes, 77454 MARNE LA VALLEE Cedex 2, FRANCE.

\section{Short title for running head:}

Benchmark solution for 3D mixed convection: part 2

\begin{abstract}
A reference solution to a benchmark problem for a 3D mixed convection flow in a horizontal rectangular channel differentially heated (Poiseuille-Rayleigh-Bénard flow) has been proposed in "Part 1: reference solution" of the present paper [Num. Heat Trans. A, vol.?, pp.?-? (2011)]. Since mixed Dirichlet and Neumann thermal boundary conditions are used on the horizontal walls of the channel, a temperature gradient discontinuity is generated. The aim of this paper is to analyze the
\end{abstract}


consequences of this singularity on Richardson extrapolation (RE) of the numerical solutions. The convergence orders of the used numerical methods (finite difference, finite volume, finite element), observed from $\mathrm{RE}$ of local and integral quantities are discussed with an emphasis on singularity influence. With the grids used, it is shown that RE can increase the accuracy of the discrete solutions, preferentially with the discretization methods of low space accuracy order, but only in some part of the channel and for a restricted range of the extrapolation coefficient. A correction to the Taylor expansion involved in the RE formalism is proposed to take into account the singularity and to explain the majority of the RE behaviors observed.

\section{Context and objectives}

To make up for the lack of numerical reference solution of the three-dimensional Navier-Stokes and energy equations for mixed convection flows, a benchmark exercise was proposed in the framework of the French Heat Transfer Society (SFT). A call for contributions was published in 2006 [1]. Two flow configurations were proposed. The first configuration is a Poiseuille-Rayleigh-Bénard (PRB) flow (i.e. a mixed convection flow in a horizontal rectangular channel heated from below) in a large aspect ratio channel at Reynolds number Re $=50$, Rayleigh number $\mathrm{Ra}=5000$ and Prandtl number $\operatorname{Pr}=0.7$. The reference solutions of this configuration have just been published in the first part of this paper [2]. These are the result of averages of the four solutions obtained by the contributors with four different solvers implemented in their own CFD research codes. Three discretization methods were used: finite difference (FD), finite volume (FV), and finite element (FE) methods. All contributors have mobilized a significant amount of computational resources to achieve reliable spatial convergence with each code. Furthermore, approximate solutions have been obtained on successively refined grids so that Richardson extrapolation (RE) could be used to extent the results. This technique is indeed known to improve the accuracy of the discrete solutions when used in the asymptotic range of the numerical methods.

However, it is mentioned in [2] that difficulties have appeared during establishing the reference solutions with RE because mixed thermal boundary conditions on the channel bottom and top plates were introduced. Indeed, in order to reproduce the operating conditions of the PRB experiments by Pabiou et al. [3], adiabatic Neumann conditions are imposed near inlet while isothermal Dirichlet conditions are imposed downstream. This choice was done to avoid a complicated mathematical formulation of the problem, using a regularizing function for instance. Therefore, in this benchmark problem, the temperature field is continuous, but the temperature gradient is discontinuous at the boundary condition junction. This type of singularity is naturally regularized when FD, FV or FE methods are used: no peculiarity is observed because the consequences of the 
singularity are generally filtered by the discretization method. However, in the framework of this benchmark exercise, we wanted to compute solutions as accurate as possible. Thus we used very fine grids and RE to try to increase the accuracy of the solutions. While the behavior of the solutions on the finest grids has remained regular, as expected, it has not been the case when RE was used. The aim of the present paper is to present and analyze these different behaviors of RE and to determine if RE can still be useful to improve the accuracy of the results in the presence of a temperature gradient discontinuity compared with the results obtained on already very fine grids. The consequences of the singularity are discussed thoroughly so that we can evaluate the degree of validity of the reference solutions proposed in [2].

One can probably wonder why such a singularity has been introduced in the benchmark problem. Singularities are sometimes encountered in benchmark problems as in the two main popular ones in convection, namely the backward facing step and the lid-driven cavity flows. In the first case, a velocity gradient discontinuity appears when the inlet channel is not considered in the computational domain and, when the inlet channel is considered, due to the reentrant corner, the derivatives of the velocity are unbounded [4]. In the lid-driven cavity case, a velocity discontinuity takes place at the two cavity corners adjacent to the driving wall. In the literature, RE and the analysis of the singularity influence are scarcely studied. Moreover, in some articles, RE is not done properly: the extrapolation is based on the formal convergence order of the used numerical method without any result on the really observed one. In the literature dealing with the liddriven cavity, one can find that it is only recently that a quasi-systematic use of RE has been proposed [5] for the main vortex characteristics and local velocities. The observed convergence order, for most of the quantities (stream function, velocity) is approximately equal to the formal one, except for the position of the vortex center, since it is a priori undetermined. Corner singularity has only a consequence on the convergence of other parameters such as the viscous drag force exerted by the fluid boundary surface [5], the enstrophy $Z$ or the palinstrophy $P[6]$. Indeed, for this problem, it is not possible to obtain convergence as there is a jump of the velocity that induces infinite derivatives. As regards the backward-facing step flow, RE does not seem to have been properly used so far, for the simplified case or the complete one, where the channel portion upstream of the step is included. Beyond providing first reference results on a 3D mixed convection problem (benchmark solution), the originality of the proposed work is to contribute to a more comprehensive analysis of $\mathrm{RE}$ in the framework of problems containing a singularity.

The outline of the paper is the following. The geometry, mathematical model and flow parameters of the simulated test case and the solvers of the different contributors are briefly presented in $\S 2$. The methodology of RE is described in $\S 3$. The fundamental assumptions for the validity of the RE technique are recalled in $\S 3.1$. The influence of the boundary condition singularity on the convergence order of RE is discussed in $§ 3.2$. 
Technical aspects used to compute reference solutions of local values by RE are given in $\S 3.3$. The results are presented and analyzed in $\S 4$. In $\S 4.1, \mathrm{RE}$ of integral values over the whole domain are discussed and used to determine the observed convergence order of our numerical methods when a singularity is present in the domain. Then, in $\S 4.2$, a selection of extrapolated local extrema of the velocity, temperature and wall Nusselt number are presented and the determination of their reference values is discussed. Some streamwise and spanwise profiles of the convergence orders observed from RE of these quantities are also discussed from the viewpoint of the singularity. Finally, in $\S 4.3$, we propose an explanation for the observed behaviors of RE and a correction to the Taylor expansion involved in the extrapolation formalism. The conclusions and the difficulties that have been raised during the study are summarized in $\S 5$.

\section{Test case and solver descriptions}

In this section, the test case geometry, the mathematical model, the parameters of the benchmark problem and the characteristics of the solvers used by the four contributors are briefly presented. The detailed description of these elements is given in the first part of this paper [2], in particular the governing equations (Navier-Stokes and energy equations with Boussinesq approximation) and the boundary conditions. The geometry and the thermal boundary conditions of the present PRB flow are given in Figure 1, where $\theta=\left(T-T_{c}\right) /\left(T_{h}-T_{c}\right)$ is the reduced temperature field. A Poiseuille flow is imposed at the channel entrance and the incoming fluid is cold $(\theta=0)$. No slip boundary conditions are imposed on the horizontal and vertical lateral walls. After an adiabatic entrance zone of streamwise aspect ratio $A_{e}$, the top horizontal wall is maintained at $\theta=0$ and the bottom wall is maintained at a higher temperature $(\theta=1) . A$ and $B$ are the streamwise and spanwise aspect ratios of the computational domain. The PRB flow is characterized by the following dimensionless parameters: $R e=50, R a=5000, \operatorname{Pr}=0.7, A=50, B=10$ and $A_{e}=2$. The resulting flow pattern is a ten longitudinal roll steady flow which is graphically presented in [2]. It is symmetrical with respect to the median longitudinal vertical plane and can therefore be computed for $y \in[0, B / 2]$.

The solvers of the four contributors are denoted by FD1, FE2, FV3 and FE4 in the present paper. FD1 is a finite difference code and FV3 is a finite volume one. Both use second order space discretization schemes. FE2 and FE4 are two finite element codes that use third order space discretization schemes. The main characteristics of the solvers of the contributors are presented in [2]. The numerical parameters used by each of these four solvers are given in Table 1 . We indicate if the symmetry with respect to the median vertical plane was used or not, the mesh sizes in each space direction, $N_{x} \times N_{y} \times N_{z}$, the time step value, $\Delta t$, an estimation of the computational time (restitution time) and the 
consistency orders ${ }^{1}, \alpha^{\circ}$, of each space discretization method for each primitive variable. Note that, when symmetry is used, $N_{y}$ is the node number on the width $B / 2$ of the computational domain.

\section{Richardson extrapolation method}

\subsection{General principle without singularity}

When the approximate solutions of a continuous initial and boundary value problem are computed by discretization methods such as FD, FV or FE methods, RE can be used to improve the precision of the discrete solutions. Indeed, provided that three main assumptions are satisfied (see below), it is possible to get an order of accuracy of at least $O\left(h^{p+1}\right)$ when the convergence order of the numerical method is $O\left(h^{p}\right)$, where $h$ is the mesh size. This technique then allows one to compute extrapolated primitive variables at any point of the computational domain as well as solution functionals such as differentiated or integrated quantities (heat and momentum fluxes, volume or surface averaged quantities, and so on). A concise and elegant presentation of RE to estimate a posteriori discretization errors in computational simulations can be found in [7]. More details and deeper discussions on the theory are given in [8, 9, 10]. Examples of extrapolated solutions in natural and mixed convection problems can be found in [11, 12].

RE first consists in computing the numerical solutions $f_{h_{i}}(1 \leq i \leq N)$ of the discretized boundary value problem on $N$ different nested uniform grids of size $h_{i}$, with $h_{1}$ the coarsest grid and $h_{N}$ the finest one. Non uniform grids with irregular distributions of the nodes are not allowed. If (assumption $\{A 1\}$ ) the exact solution of the continuous problem, $f_{\text {exact }}$, is sufficiently smooth to justify the use of Taylor expansion (at least up to the discretization order), then it can be written in the form:

$$
f_{h_{i}}=f_{\text {exact }}+C_{\alpha} h_{i}^{\alpha}+O\left(h_{i}^{\alpha+1}\right)
$$

where $C_{\alpha}$ is a coefficient which is dependent on $\alpha$ but independent of $h_{i}$. Then, the leading order $\alpha$ of the truncation error due to discretization, the coefficient $C_{\alpha}$ and the exact solution $f_{\text {exact }}$ can be approximated from the discrete solutions, if two more assumptions are satisfied. The second assumption $\{A 2\}$ is that the mesh spacings $h_{i}$ used in the extrapolation must be small enough so that the discrete solutions $f_{h_{i}}$ are located in the asymptotic convergence region. In other words, the leading order term $C_{\alpha} h_{i}^{\alpha}$ of the truncation error must truly dominate the total discretization error $f_{\text {exact }}-f_{h_{i}}$. In this case, $\alpha$ will be considered as the observed convergence order from RE.

\footnotetext{
${ }^{1}$ the consistency order is the formal convergence order that is the leading order of the space discretization truncation error.
} 
Thus, using three grids $(N=3)$ such as $\frac{h_{1}}{h_{2}}=\frac{h_{2}}{h_{3}}$, the approximations $\tilde{\alpha}, \tilde{C}_{\alpha}$ and $\tilde{f}^{e x}$ of $\alpha, C_{\alpha}$ and $f_{\text {exact }}$ in equation (1) are given by $[9,10]$ :

$$
\begin{aligned}
\tilde{\alpha} & =\frac{\ln \left(\frac{f_{h_{1}}-f_{h_{2}}}{f_{h_{2}}-f_{h_{3}}}\right)}{\ln \left(\frac{h_{1}}{h_{2}}\right)} \\
\tilde{C}_{\alpha} & =\frac{f_{h_{2}}-f_{h_{3}}}{h_{2}^{\tilde{\alpha}}-h_{3}^{\tilde{\alpha}}} \\
\tilde{f}^{e x} & =f_{h_{3}}-\tilde{C}_{\alpha} h_{3}^{\tilde{\alpha}}
\end{aligned}
$$

and, using four grids $(N=4)$ such as $\frac{h_{1}}{h_{2}}=\frac{h_{3}}{h_{4}}$, they are given by:

$$
\begin{aligned}
\tilde{\alpha} & =\frac{\ln \left(\frac{f_{h_{1}}-f_{h_{3}}}{f_{h_{2}}-f_{h_{4}}}\right)}{\ln \left(\frac{h_{1}}{h_{2}}\right)} \\
\tilde{C}_{\alpha} & =\frac{f_{h_{3}}-f_{h_{4}}}{h_{3}^{\tilde{\alpha}}-h_{4}^{\tilde{\alpha}}} \\
\tilde{f}^{e x} & =f_{h_{4}}-\tilde{C}_{\alpha} h_{4}^{\tilde{\alpha}}
\end{aligned}
$$

with $\tilde{C}_{\alpha}=C_{\alpha}+O\left(h_{N-1}\right)$ and $\tilde{f}^{e x}=f_{\text {exact }}+O\left(h_{N}^{\tilde{\alpha}+1}\right)$. As a consequence, the approximation $\tilde{f}^{e x}$ will be closer to the asymptotic solution $f_{\text {exact }}$ with decreasing $h_{N}$ and increasing $\tilde{\alpha}$. Thereafter, $\tilde{\alpha}$ and $\tilde{f}^{e x}$ will respectively be noted $\alpha$ and $f^{e x}$.

The formal expression of the Taylor expansion (1) is valid for multidimensional problems, in any coordinates, including space and time, only if (assumption $\{A 3\})$ the same grid refinement ratio is applied in all space and time directions. In our stationary problem, this means that the cell aspect ratios are kept constant from one grid to another. That is, if $N$ uniform Cartesian grids of size $\Delta x_{i}, \Delta y_{i}$ and $\Delta z_{i}(i=1, \ldots, N)$ are used for $\mathrm{RE}$, the ratios $\frac{\Delta x_{i}}{\Delta z_{i}}$ must be equal whatever $i$, and the same holds for $\frac{\Delta y_{i}}{\Delta z_{i}}[8,9,11]$.

On smooth problems, the spatial convergence orders of the codes used to compute the FD1 and FV3 solutions have been shown to be equal to two (see [13] for the FD1 code) while, for the two FE codes FE2 and FE4, they have been shown to be equal to three for the temperature $\theta$ and the velocity components $u, v, w$. That is, for the four codes used in the present study, the spatial convergence order for $u, v, w$ and $\theta$ is equal to the consistency order, $\alpha^{\circ}$, mentioned in Table 1 . As a consequence, if the solution $f_{\text {exact }}$ of the problem is smooth enough (say $f_{\text {exact }} \in C^{2}(\Omega \cup \partial \Omega)$, where $\Omega \in \mathbb{R}^{3}$ is the computational domain and $\partial \Omega \in \mathbb{R}^{2}$ its boundary), the $u, v, w$ and $\theta$ values that will be given as reference solutions from RE should only be obtained with an associated extrapolation coefficient $\alpha$ equal to two for the FD1 and FV3 solutions and to three for the FE2 and FE4 solutions. Otherwise, if $\alpha$ is very different from $\alpha^{\circ}$, this means that the discrete solutions used to compute the extrapolated solution are not in the asymptotic convergence region of $\mathrm{RE}$ (assumption $\left\{A_{2}\right\}$ is not satisfied) or that the solution of the continuous problem is not 
smooth enough (assumption $\{A 1\}$ is not satisfied). This is precisely what is observed in the present problem and what is developed in the following subsection.

\subsection{Singularity influence on the RE convergence orders}

The solution of the present problem is not smooth because the temperature gradient on the horizontal plates is discontinuous at $x=0$. This is due to the use of homogeneous Neumann (adiabatic) conditions for $-2 \leq x<0$ and Dirichlet boundary conditions for $x \geq 0$. To explain the temperature gradient discontinuity, let us consider the isotherms near this singular point: the left-hand isotherms tend to be perpendicular to the wall due to the homogeneous Neumann condition while the right-hand isotherms tend to be parallel to the wall due to the constant Dirichlet condition. With such a change in the thermal boundary conditions, to avoid any singularity, a wall with a convex $90^{\circ}$ step would be necessary.

To understand the effect of this singularity on the convergence rate of the solutions and, as a consequence, on the convergence rates, $\alpha$, associated with $\mathrm{RE}$, let us refer to the FE framework. Without any singularity, the theoretical FE discretization error of an elliptic problem is given by the basic interpolation theory to be $O\left(h^{\alpha^{\circ}}\right)$ in the $L^{2}$ norm, where the consistency order of the discretization method is given by $\alpha^{\circ}=k+1$, with $k$ being the polynomial approximation degree and $h$ the characteristic mesh size $(k=2$ and $\alpha^{\circ}=3$ for $\theta, u, v$ and $w$ in the FE2 and FE4 methods). But, as soon as a singularity is present, the rate of convergence of the numerical model becomes $O\left(h^{\min \left(\alpha^{\circ}, r\right)}\right)$, where $r$ measures the problem regularity influence on the actual convergence rate (see [14], section 14, p. 404). It seems therefore reasonable to consider that the convergence rates, $\alpha$, obtained from RE of integral quantities based on a norm should be equal to $\min \left(\alpha^{\circ}, r\right)$, if the grids are located in the asymptotic convergence regions of the discrete solutions. However, what happens with RE of other quantities, such as local extrema for instance? Does $\alpha$ vary between $\min \left(\alpha^{\circ}, r\right)$ and $\max \left(\alpha^{\circ}, r\right)$ ? Does the singularity only influence its neighborhood or the whole domain? These issues will be dealt with in $\S 4$.

Strang and Fix, in chapter 8 "Singularities" of reference [4], propose a theoretical analysis to a priori determine the regularity $r$ of a singular boundary value problem due to the discontinuity of its boundary conditions. They analyze the singularity influence on the convergence rate of FE methods. Their analysis focuses on a test case corresponding to the displacement computation in a 2D domain with a crack. As shown in Figure 2, since the crack axis is a symmetrical axis, this test case can be viewed as a 2D pure diffusion problem (Poisson problem) with a mixed Dirichlet/Neumann condition on one of its boundaries. We can see that this test case presents the same singularity as in the present benchmark problem except of its two-dimensionality (the benchmark problem is 3D) and of absence of convection. Strang and Fix [4] showed that the solution around 
the singularity behaves like $x^{1 / 2}$, where $x$ is the distance to the singularity, and that the error of the FE solutions of this elliptic problem is $O(h)$ for any choice of element. Thus, the problem regularity is $r=1$. But they also mentioned that away from the singularity a higher regularity can be expected due to a decrease of the singularity pollution. In the present benchmark exercise, the determination of the problem regularity will be done a posteriori in $\S 4.1 .2$ by performing specific numerical spatial convergence studies. We will show, as in Strang and Fix [4], that the regularity $r$ of the solutions attached to the thermal field tends to 1 near the singularity and increases up to $\alpha^{\circ}$ away from it.

\subsection{Technical aspects of Richardson extrapolation of local values}

Grid doubling or integer grid refinement ratios are not required for the validity of RE. Thus, in the general case, solutions of the coarsest grids are not computed at the nodes of the finest grid. However, if local values have to be evaluated by RE, it is necessary to have the values of $f_{h_{i}}$ at the same points in equations (2) and (3). As suggested by Roache [8], this is made possible by building an interpolation of the coarse solutions on the finest grid, the order of which is higher than the space discretization order of the used numerical method.

Since the consistency order, $\alpha^{\circ}$, of the numerical methods used in the present paper is equal to two or three (see Table 1), a cubic spline interpolation has been used to compute the solutions of the coarsest grids at the nodes of the finest one, before doing RE of the temperature, Nusselt number and velocity local extrema (see §4.2.3). Indeed, cubic spline interpolation is supposed to be third order if the solution is smooth enough and the points far enough from the boundaries. Other interpolation methods could have been used: for instance, an evaluation of the influence of Lagrangian interpolations on $\mathrm{RE}$ is proposed in [15]. In the present paper, the values and the coordinates of the local extrema of the extrapolated solution are also computed using cubic spline interpolation between the finest grid nodes. This is illustrated in Figure 3 in which a zoom in the $w$ streamwise profiles computed with the four grids of FD1, together with the extrapolated profiles, are presented. This figure allows to determine the FD1 value and the streamwise coordinate of the vertical velocity local extremum noted $w_{2}$ and $x_{2}$ in Table 3.

\section{Result presentation and analysis}

\subsection{Richardson extrapolation of integral quantities}

\subsubsection{Presentation and discussion of the results}

The extrapolated values of integral quantities are computed first. These integrals are: the mean kinetic energy multiplied by two, $2 E_{c}$, on the whole domain of volume $D$, the 
mean pressure difference, $\Delta P_{i o}$, between inlet surface $\left(S_{i}\right)$ and outlet surface $\left(S_{o}\right)$ and the mean temperature, $T_{m}$, on the whole domain that are defined by:

$$
\begin{gathered}
2 E_{c}=\frac{1}{D} \iiint_{D}\left(u^{2}+v^{2}+w^{2}\right) d x d y d z \\
\Delta P_{i o}=\frac{1}{S_{i}} \iint_{S_{i}} P d y d z-\frac{1}{S_{o}} \iint_{S_{o}} P d y d z \\
T_{m}=\frac{1}{D} \iiint_{D} \theta d x d y d z
\end{gathered}
$$

These values have been computed using either the middle point rule for the FD1 and FV3 solutions or $3 \times 3 \times 3$ Gauss integration scheme for the FE2 and FE4 solutions. The advantage of these integrals is that they depend only on the primitive variables: no differentiation and no interpolation are needed to compute their values on each grid. Thus, in this case, the validity of $\mathrm{RE}$ and the values of the associated extrapolation coefficient, $\alpha_{E_{c}}, \alpha_{\Delta P_{i o}}$ and $\alpha_{T_{m}}$, depend only on the convergence orders of the numerical methods and on the satisfaction of the three assumptions $\{A 1\}$ to $\{A 3\}$ stated in $\S 3.1$.

The values of $f=\left(2 E_{c}, \Delta P_{i o}, T_{m}\right)$ on the finest grid (noted $f^{f g}$ ) and extrapolated by $\mathrm{RE}$ (noted $f^{e x}$ ) are given in Table 2 with the associated convergence order, $\alpha_{f}$, and with the relative distance between the finest grid and extrapolated solutions: $d_{f}=\left(f^{f g}-\right.$ $\left.f^{e x}\right) /\left|f^{f g}\right|$. It has been checked in [16] that the asymptotic convergence region is not reached by the FE4 solutions for $\Delta P_{i o}$ and $2 E_{c}$. Therefore RE cannot be used for these FE4 quantities. Note however that $2 E_{c}^{f g}$ and $\Delta P_{i o}^{f g}$ values on the FE4 finest grid are very close to the extrapolated values $2 E_{c}^{e x}$ and $\Delta P_{i o}^{e x}$ of the three other contributors: comparing with the other contributors values, the accuracy of the values on the FE4 finest grid are satisfactory without requiring RE.

To give a quick and simple means of comparing the values obtained by the four contributors for each quantity presented in this paper, we have computed the arithmetic mean of the extrapolated or finest grid values and the maximum discrepancy between them. For a given quantity, we have considered that its reference value is this mean and that the uncertainty margin on the reference value is the maximum discrepancy between the values of the four contributors. More precisely, in the case of the integral quantities computed in Table 2 , the reference value, $f_{\text {ref }}$, is equal to the arithmetic average of the extreme values of the FD1, FE2 and FV3 extrapolated values, plus the extrapolated or the finest grid FE4 value depending on whether RE succeeds or not. The uncertainty margin, $f_{\text {marg }}$, is equal to the half difference between the two extreme values. The precision of the determination, $f_{\text {prec }}$, is equal to the ratio of the uncertainty margin to the reference value. The reference values of the integral quantities are given in Table 2. The obtained precisions are of the order of $10^{-5}$, with five common figures among the four solutions for $2 E_{c}$ and $\Delta P_{i o}$ and four common figures for $T_{m}$. 
It can also be noted that the relative distances, $d_{f}$, between the finest grid solutions and the extrapolated solutions (when admissible) are higher for the second order methods (FD1 and FV3) than for the third order methods (FE2 and FE4). All these relative distances are higher than the precision of the determination of the reference values (except for $2 E_{c}$ in FE2 column), showing that RE improves the accuracy of the reference values, more substantially for the second order methods than for the third order ones. It should also be added that, for the FE4 contribution, the maximum relative distance on $2 E_{c}$ (resp. $\left.\Delta P_{i o}\right)$ between the coarsest and finest grids are equal to $4.25 \times 10^{-6}$ (resp. $1.45 \times 10^{-5}$ ), which is smaller than the precision of the reference values given in Table 2 . It therefore confirms that RE is useless for the FE4 method to get the same accuracy as the other methods for these particular quantities.

Finally, in Table 2, one can see that the coefficients $\alpha_{E_{c}}$ and $\alpha_{\Delta P_{i o}}$ obtained when $\mathrm{RE}$ is admissible, are respectively nearly equal to the consistency orders $\alpha_{u, v, w}{ }^{\circ}$ and $\alpha_{p}{ }^{\circ}$ of each numerical method (see Table 1). This corresponds to the expected behavior for a smooth problem without discontinuity (see $\S 3.1$ ). On the other hand, $\alpha_{T_{m}}$ is nearly equal to one for the four contributions despite $\alpha_{\theta}{ }^{\circ}=2$ for the FD1 and FV3 solutions and $\alpha_{\theta}{ }^{\circ}=3$ for the FE2 and FE4 solutions. In the next paragraph, we are going to show that the lower than expected $\alpha_{T_{m}}$ values are due to the singularity induced by the thermal boundary conditions.

\subsubsection{Determination of the singularity influence}

To analyze the influence zone of the singularity of the present benchmark problem, extrapolated values of the mean temperature $T_{m}$ (see equation (6)) and of the $L^{2}$ norms of temperature, $T_{L^{2}}$, and vertical velocity component, $W_{L^{2}}$, have been obtained by $\mathrm{RE}$ in several subdomains of the whole computational domain. Here the $L^{2}$ norm is defined by $f_{L^{2}}=\left(\iiint_{D} f^{2} d x d y d z\right)^{1 / 2}$. In all the subdomains considered, the extension is maximum in $y$ and $z$ transverse directions (that is $0 \leq y \leq B / 2$ or $B$ depending on the contributor and $0 \leq z \leq 1$ ) and it varies from $x=-2$ to $x^{\circ}$ for the upstream subdomains and from $x=x^{\circ}$ to $A=48$ for the downstream subdomains, with $-2<x^{\circ}<48$. Then the convergence orders $\alpha_{T_{m}}, \alpha_{T_{L^{2}}}$ and $\alpha_{W_{L^{2}}}$, observed from $\mathrm{RE}$ of $T_{m}, T_{L^{2}}$ and $W_{L^{2}}$ respectively, are computed in the downstream and upstream subdomains. The profiles of some of these quantities are drawn as a function of $x^{\circ}$ in Figures $4(a-b)$ (the whole profiles can be found in [16]).

Figures $4(\mathrm{a}-\mathrm{b})$ show that $\mathrm{RE}$ of $T_{m}, T_{L^{2}}$ or $T_{W^{2}}$ succeeds, except locally for the FD1 $\alpha_{T_{m}}$ value in the upstream subdomain $\left(\alpha_{T_{m}}=10.2\right.$ at $\left.x^{\circ}=-1.75\right)$ and for the FE2 $\alpha_{W_{L^{2}}}$ value in the downstream subdomains near $x^{\circ}=15$. In Figure 4(a), it appears that $\alpha_{T_{m}} \rightarrow 1$ for the upstream subdomains located near the beginning of the heated plate $\left(x^{\circ}=0\right)$, and $\alpha_{T_{m}}$ increases when the subdomain locations move farther downstream: for the FD1 
solution, the $\alpha_{T_{m}}$ values on the downstream subdomains stabilize around 1.8 (that is around the consistency order $\alpha^{\circ}=2$ ) for $20 \leq x^{\circ} \leq 48$, while it increases beyond $\alpha^{\circ}=3$ for the two FE solutions. The behavior of $\alpha_{T_{L^{2}}}$ for the FV3 solution is similar to the behavior of $\alpha_{T_{m}}$ for the FD1 solution: $\alpha_{T_{L^{2}}}$ is nearly equal to one near $x^{\circ}=0$ for the upstream subdomains $\left(\alpha_{T_{L^{2}}} \approx 1.3\right.$ for $\left.x^{\circ}=0\right)$ and it increases towards $\alpha^{\circ}$ in the downstream subdomains $\left(\alpha_{T_{L^{2}}} \approx 1.7\right.$ for $\left.x^{\circ}>20\right)$. The slight differences between the FV3 solution and the other solutions are maybe due to the different discretization schemes used by the contributors for the convective term of the energy equation: centered difference schemes for the FD1, FE2 and FE4 solutions and Quick scheme for the FV3 solution. It can therefore be considered that the influence zone of the thermal boundary condition singularity on the convergence orders of the quantities linked to temperature extends to $x=20$ to 25 in the present PRB flow, regardless of the numerical method used. As a conclusion of this analysis, it appears that the effect of the singularity of the present problem spatially varies: it diminishes with the distance to the singularity. Furthermore the exponent $r$ introduced in $\S 3.2$ to characterize the regularity of the solution tends to 1 , regardless of the numerical method used, at least in the neighborhood of the upstream edge of the heated zone and for the quantities derived from the temperature field.

The dynamics fields should also be affected by this singularity through the buoyancy term in the momentum equation which makes a coupling of the velocity and temperature fields. To study this influence, $\mathrm{RE}$ of $W_{L^{2}}$ in the downstream subdomains is presented in Figure 4(b). RE of $W_{L^{2}}$ succeeds, except for the FE2 solution for $x^{\circ}<20$. It appears that $\alpha_{W_{L^{2}}} \approx 2=\alpha^{\circ}$ for the FD1 and FV3 solutions for any $x^{\circ}$, whereas $\alpha_{W_{L^{2}}} \approx 1.4$ near inlet for the FE4 solution, and $\alpha_{W_{L^{2}}}$ tends to values that vary between 3 and 5 for $x^{\circ}>20$ for the two FE solutions. Thus, the singularity of the thermal boundary conditions does not seem to affect the velocity field with the second order FD1 and FV3 methods for the used grids, whereas it influences the velocity field with the third order FE2 and FE4 methods. In the latter case, the length of the influence zone of the singularity is the same as for the temperature integrals in Figures $4(\mathrm{a})$ : it reaches $x^{\circ} \approx 25$.

To sum up the above observations, it appears that the RE behavior greatly varies and that the singularity has not the same influence according to the accuracy order of the discretization method. The variation range of the extrapolation coefficient $\alpha_{f}$ seems much larger with the third order methods. It also appears that the grid levels that correspond to the asymptotic convergence region for the second order methods might not correspond to the asymptotic region of the higher order methods. An explanation of these very different behaviors of RE is proposed in $\S 4.2 .2$ and $\S 4.3$.

As a consequence of the above observations, we have considered that the reference quantities defined in the present benchmark problem should be established from the extrapolated quantities only if the associated convergence rates $\alpha$ are such as $1 \leq \alpha \leq \alpha^{\circ}$. In practice, we have taken into account numerical errors by using superior tolerance 
margins on $\alpha$ to choose the conserved extrapolated values: in this paper, the reference solutions are defined from the extrapolated solutions with $1 \leq \alpha \lesssim 2.5$ for the FD1 and FV3 contributions and with $1 \leq \alpha \lesssim 4$ for the FE2 and FE4 contributions.

\subsection{Richardson extrapolation of temperature, velocity and Nus- selt number local extrema}

\subsubsection{Space profiles of the thermal and dynamical fields}

In the following, we denote by $N u_{t}$ and $N u_{b}$ the local Nusselt numbers on the top and bottom walls respectively. They are defined by:

$$
N u_{t, b}(x, y)=-\frac{H\left(\frac{\partial T}{\partial Z}\right)_{Z=H, Z=0}}{T_{h}-T_{c}}=-\left(\frac{\partial \theta}{\partial z}\right)_{z=1, z=0}
$$

In the variational context of FE methods, it is possible to compute the Nusselt numbers in several ways. The "non consistent" way simply uses the definition (7), i.e. the $z$-derivative of the interpolation function for $\theta$ is computed. The "consistent" way exploits the duality between Dirichlet and Neumann boundary condition. The "consistent" flux at a Dirichlet boundary node is the one that would yield the same solution if prescribed instead of the Dirichlet condition. Details on how to compute such a flux in a FE framework are given in references $[17,18]$. A reported advantage of the "consistent" flux is that it is generally more precise than the non-consistent one. This is also what we have observed here (see §4.2.3). In the present study, the FE2 Nusselt numbers are the non consistent ones while the FE4 contribution proposes the two Nusselt number types. The consistent Nusselt numbers will be denoted by $N u_{t}^{\text {cons }}$ and $N u_{b}^{\text {cons }}$ while the notations $N u_{t}$ and $N u_{b}$ will be kept to denote the non consistent Nusselt numbers and to denote the Nusselt numbers in a generic way.

In Figures 3, 4 and 5 of the first part of this paper [2], several longitudinal and transversal profiles of the primitive variables $\theta, u, v$ and $w$ and of the Nusselt numbers $N u_{t}$ and $N u_{b}$ are drawn along straight lines into the computational domain. Here, in $\S 4.2 .3$, we are interested in determining the reference values of some local extrema on these profiles.

Note that it is not possible to draw the extrapolated profiles (from RE) of the primitive variables or Nusselt numbers in the present problem because it is impossible to be located in the asymptotic convergence region along the whole profiles. In particular, RE diverges at points where the profiles computed on two distinct meshes intersect. Indeed, when $f_{h_{i}}=f_{h_{j}}$ for $h_{i} \neq h_{j}, \alpha$ diverges in equations (2) or (3). This is illustrated in Figure 5 that focuses on a zone where a curve crossing is present. Such behaviors are also observed in [19]. 


\subsubsection{Space profiles of the observed convergence rates from $\mathrm{RE}$}

To complete the last observation on the curve crossings, a selection of streamwise and spanwise profiles of the convergence rates, $\alpha$, observed from RE of the different primitive variables and Nusselt numbers are presented in Figures 6 and 7. To avoid overloading the figures, only one FE profile is presented among the FE2 and FE4 solutions because the $\alpha$ profiles computed by the two FE codes are very similar. In particular, they diverge nearly at the same points. All the $\alpha$ profiles of the four contributors are available in [16].

One can see that the $\alpha$ profiles show chaotic behavior and that RE can even fail. This happens when the values of the studied quantity do not monotonously vary from one grid to the following finer one. This behavior is indicated by arbitrarily fixing $\alpha$ to zero in some profiles. As already seen in Figure 5, $\alpha$ profiles present several sharp overshoots and undershoots at the points where the field profiles on the different grids intersect [19]. This is the case for instance at $x=0$ for all the variables of the four contributions, but also in nearly all the entrance zone for the FD1, FE2 and FE4 contributions. This is due to the probable conjunction of two causes. First, the exact solution of the cold Poiseuille flow imposed as inlet boundary condition at $x=-2$ is nearly conserved until the beginning of the heated plate at $x=0$ on all the grids. Second, the FD1, FE2 and FE4 contributions use centered discretization schemes for the convective terms and very small oscillations are observed in their temperature and velocity streamwise profiles around $x=0$ with their coarsest grids such as $N_{x} \leq 601$ (more precisely, no velocity oscillation is observed in the FD1 solution and very small velocity oscillations are observed on all the grids of the FE4 solution). These oscillations generally appear just around $x=0$ because a streamwise acceleration of the flow due to the density variation near the bottom plate and high transverse thermal gradients are present at the same place. No oscillations are observed in the FV3 solutions because the Quick scheme is used.

Generally, the FD1 and FV3 $\alpha$ profiles are much more regular than the FE2 and FE4 ones. The FD1 and FV3 $\alpha$ values for $\theta, u, v, w$ are nearly equal to $\alpha^{\circ}=2$ in the downstream zone for $x>20$. On the other hand, the FE4 and FE2 RE can fail, even in the downstream zone, or can succeed but with associated $\alpha$ values very different from $\alpha^{\circ}=3$. This is probably due to the higher precision of the FE methods used here. Indeed, the values computed on each grid with these methods are very close to each other. For instance, the maximum relative distance computed for the primitive variables between the coarsest and the finest grid solutions of the FE2 and FE4 contributions generally varies between $10^{-4}$ and $10^{-5}$ (or even less) where it varies between $10^{-2}$ and $10^{-3}$ for the FD1 and FV3 solutions. As a consequence, the FE solutions are very sensitive to the numerical errors, to the entrance singularity and to the curve crossings. A way of limiting these negative effects on the RE with the high order methods would have been to increase the size ratio of the successive grids. However this solution has appeared impossible in 
the present case due to the computational costs on grids much finer than those already used.

Finally, for the four contributions, the $\alpha$ values associated with $\theta$ and $N u_{t}$ are generally smaller than $\alpha^{\circ}$ for $x<20$, except where over- and undershoots are present. These values approach 1 for $x<10$ due to the singularity influence (cf. §4.1.2). The $\alpha$ values associated with $N u_{t}$ also vary between 1 and $\alpha^{\circ}$ in the spanwise direction (see Figure 7(c)). This explains why $\alpha \approx 1.2$ for $x>20$ in the FV3 $N u_{t}$ profile at $(y, z)=(2,1)$ (see Figure $7(\mathrm{a})$ ) and $\alpha \approx 1.7$ for $x>25$ in the FE4 (and FE2) $N u_{t}$ profiles at $(y, z)=(5,1)$ (see Figure $7(b))$.

\subsubsection{Temperature, velocity and Nusselt number local extrema}

As it has just been discussed, extrapolated solutions of the present problem cannot be determined for the whole field but only for some local values, such as local extrema, or for integral quantities (see $\S 4.1 .1$ for instance). The extrapolated values and the coordinates of eighteen local extrema of $\theta, u, v, w, N u_{t}$ and $N u_{b}$ have been computed using the method described in §3.3. These extrema are graphically presented in the Figures 3, 4 and 5 in [2]. Their values are given in Tables 3-5 of the present paper (the extrapolated values of other local extrema and of their coordinates are given in $[16,2])$.

For the FD1, FE2 and FV3 solutions, more than $70 \%$ of the whole extrema that have been computed during this study (more than one hundred in total) have been extrapolated with an associated extrapolation coefficient, $\alpha$, whose value is equal to $\alpha^{\circ} \pm 20 \%$, that is very close to the spatial consistency order, $\alpha^{\circ}$, of the numerical method used. For the other extrema, $\alpha$ values do not agree with the consistency order for the various reasons already listed above: intersection of the profiles computed on the different grids, influence zone of the thermal boundary condition singularity for $x<20$ and, probably, influence of the boundary conditions on the cubic spline interpolations when the extrema are very close to the wall. For the FE4 solutions, the $\alpha$ values are very different of $\alpha^{\circ}$ for the majority of the extrema. FE4 RE might fail because its second assumption $\{$ A2 $\}$ is not satisfied (the discrete solutions are not located in the asymptotic convergence region), although $\{$ A2\} is satisfied for the other methods. Subsection $\S 4.3$ elaborates on this point and proposes further explanations.

A part of the extrapolated values and coordinates of the local extrema of the primitive variables for which $1.2<\alpha<2.1$ for the FD1 and FV3 solutions, and $1.1<\alpha<4.5$ for the FE2 and FE4 solutions are given in Tables 3-4, together with the associated $\alpha$ values and the relative distance $d$ between the extrapolated and the finest grid values. In several cases (mainly concerning the FE4 solutions), only the values and the coordinates on the finest grid are provided because the associated $\alpha$ values are too high or too small compared with the $\alpha$ ranges given above. In these cases, the $\alpha$ values are replaced by 
$* * *$.

Reference solutions for the local extrema and their coordinates have been determined in the same way as those of the integral values presented in §4.1.1. These reference solutions (denoted by $f_{r e f}, x_{r e f}$ or $y_{r e f}$ ), with their uncertainty margin (denoted by $f_{\text {marg }}$, $x_{\text {marg }}$ or $y_{\text {marg }}$ ) and their precision (denoted by $f_{\text {prec }}$ ) are given in Tables $3-4$. Here, the reference values are equal to the arithmetic average of the minimum and maximum values of the extrapolated values of the four contributors, except when the FE2 or FE4 RE fails. In this case, the FE2 or FE4 extrapolated value is replaced by the FE2 or FE4 finest grid value.

On the other hand, the FD1 and FV3 solutions on the finest grid are never used to define the reference solutions. Indeed, as it can be seen in Table 3 , the relative distances $d$ on $\theta, u, v, w$ for the FD1 and FV3 solutions are nearly always one or two orders higher than $f_{\text {prec }}$ whereas, for the FE2 and FE4 solutions, they are always of the same order or smaller than $f_{\text {prec }}$ (when $\mathrm{RE}$ is possible). This means that, with the definition and the precision of the reference values given here, $\mathrm{RE}$ of the discrete solutions obtained by the FE methods are useless to determine the reference values of $\theta, u, v, w$. RE is useful only to allow for the second order FD1 and FV3 methods to give solutions with a third order precision equal to the precision of the FE2 and FE4 methods.

The same observation as for the primitive variables can be done with the consistent Nusselt numbers $N u_{t}^{\text {cons }}$ and $N u_{b}^{\text {cons }}$ computed with the FE4 method. That is why only their values on the finest grid are proposed in Table 5. On the other hand, RE is useful to determine the reference values from the non consistent Nusselt numbers (compare $d$ and $f_{\text {prec }}$ in Table 5 for instance). The extrapolated values of the non consistent Nusselt numbers and the finest grid values of the consistent Nusselt numbers are thus kept to define the reference values of the Nusselt numbers.

Following the methodology just described, the reference values of all the primitive variable local extrema that have been computed in this benchmark exercise can be given with four to five significant figures and those of the Nusselt number with three to four significant figures. Their coordinates can generally be given with three significant figures in $x$ direction and with four significant figures in $y$ direction.

\subsection{Explanation of the observed behaviors of Richardson extrap- olation}

The preceding sections have shown a variety of behavior when trying to apply Richardson extrapolation: (i) working behavior with an observed convergence order $\alpha$ equal to the consistency order $\alpha^{\circ}$ of the discretization method; (ii) working behavior with an observed convergence order $\alpha$ between 1 and $\alpha^{\circ}$; (iii) non-working behavior. In this section, we discuss these observations. The basic idea is to assume that the approximation error 
to a quantity $f_{h}$ can be written as two main terms within the Taylor expansion instead of one as in equation (1), section 3.1. Namely :

$$
f_{h}\left(h, C_{\alpha^{\circ}}, \alpha^{\circ}, C_{r}, r\right)=f_{\text {exact }}+C_{\alpha^{\circ}} h^{\alpha^{\circ}}+C_{r} h^{r}+O\left(h^{1+\max \left(\alpha^{\circ}, r\right)}\right)
$$

where $C_{\alpha^{\circ}} h^{\alpha^{\circ}}$ is the leading term of the approximation error to the regular part of the solution (same term as the one in equation (1)) and $C_{r} h^{r}$ is the leading term of the approximation error to the singular part of the solution. As before $\alpha^{\circ}$ is the consistency order of the discretization and $r$ measures the problem regularity influence on the actual convergence rate. Here $f_{h} \rightarrow f_{\text {exact }}$ when $h \rightarrow 0$.

When $h \rightarrow 0$, the term with largest exponent becomes negligible and Richardson extrapolation allows us to determine the smallest exponent and associated constant $C$ as in section 3.1. However, in practice, we work with a fixed sequence of 3 (or 4 ) given $h$ values, say $\left\{h_{1} ; h_{2} ; h_{3}\right\}=\left\{h_{1} ; \frac{h_{1}}{\tau} ; \frac{h_{1}}{\tau^{2}}\right\}$ with $\tau>1$. Scaling equation (8) with $\tilde{f}_{h}=\frac{f_{h}}{f_{\text {exact }}}$ and $\tilde{h}=\frac{h}{h_{1}}$, one gets:

$$
\tilde{f}_{h}\left(\tilde{h}, \tilde{C}_{\alpha^{\circ}}, \alpha^{\circ}, \tilde{C}_{r}, r\right)=1+\tilde{C}_{\alpha^{\circ}} \tilde{h}^{\alpha^{\circ}}+\tilde{C}_{r} \tilde{h}^{r}+O\left(\tilde{h}^{1+\max \left(\alpha^{\circ}, r\right)}\right)
$$

In the following, we use the scaled equation (9), leaving out the tildes on $\tilde{f}, \tilde{h}, \tilde{C}_{\alpha^{\circ}}$ and $\tilde{C}_{r}$ for notation clarity. For example, our fixed sequence of (scaled) $h$, is now: $\left\{1 ; \frac{1}{\tau} ; \frac{1}{\tau^{2}}\right\}$. Then we choose typical numerical values $C_{\alpha^{\circ}}=10^{-4}<<1, \tau=2$ and $r=1$ and we plot the observed convergence order $\alpha$ as a function of the ratio $\rho=\frac{C_{r}}{C_{\alpha^{\circ}}}$ when we apply the RE process (equation (2) of section 3.1) to our model function $f_{h}$ (equation (9)), neglecting the $O\left(h^{1+\max \left(\alpha^{\circ}, r\right)}\right)$ term, in the four following cases : (i) $\rho>0, \alpha^{\circ}=2$; (ii) $\rho>0, \alpha^{\circ}=3$; (iii) $\rho<0, \alpha^{\circ}=2$; (iv) $\rho<0, \alpha^{\circ}=3$.

We also define a Richardson efficiency ratio $\sigma$ as follows:

$$
\sigma=\log \frac{\left|f^{e x}-f_{\text {exact }}\right|}{\max \left(\left|C_{\alpha^{\circ}} h_{3}^{\alpha^{\circ}}\right|,\left|C_{r} h_{3}^{r}\right|\right)}
$$

where $f^{e x}$ is the extrapolated function. If $\sigma<0$, this means that RE has been successful in reducing the main component of the error compared to its value for the smallest $h$.

Figure 8(a) (resp. Figure 8(b)) shows the profile of $\alpha$ and $\sigma$ as a function of $\log |\rho|$ when $\rho>0$ (resp. $\rho<0$ ). On the two plots, we can distinguish three zones:

Zone $1 \log |\rho| \lesssim-1$ where the approximation error of the regular part of the solution dominates the approximation error of the singular part;

Zone $2-1 \lesssim \log |\rho| \lesssim 1$ where the approximation error of the regular and singular parts of the solution have the same order of magnitude;

Zone $31 \lesssim \log |\rho|$ where the approximation error of the singular part of the solution 
dominates. This zone corresponds to the asymptotic range in the present example since $r=1$ and $\alpha^{\circ}=2$ or 3 .

We can make the following remarks:

1. Zone 1 and 3 are the zones where RE is effective in reducing the error. This was expected for Zone 3 which is in the asymptotic range as defined in section 4.1, but not necessarily so for Zone 1;

2. In Zone 2, the behavior of RE depends on the sign of $\rho$ : when $\rho<0$, RE is not applicable, while if $\rho>0$, RE still gives a result. However, as the profile of $\sigma$ on Figure 8 (a) shows, very little improvement in the reduction of the error is to be expected. We can conclude by saying that in Zone 2, RE is not very useful;

3. For the third-order methods, the interval of $\log |\rho|$ on which RE doesn't bring significant error reduction (say $|\sigma|>-0.5$ ) is larger than for second-order methods by one magnitude order;

4. As shown by the $\sigma$ profiles, RE is less efficient at reducing the error for third-order methods than for second-order methods.

Remark 1 is consistent with our observations of the second order methods FD1 and FV3 for which RE seems to improve the results even though we are not in the asymptotic range. Therefore, most quantities seem to behave as if in Zone 1, with the notable exception of the mean temperature.

Remark 3 is related to the fact that, for FE4 and FE2, RE was found to be much more difficult to apply than for the low order methods: this suggests that most quantities behave as if in Zone 2. This fact can be tempered with Remark 4 which suggests that less improvement in the error is to be expected for third order method than for second order method.

Applicability of RE was found to be better for FE2 than for FE4: Remark 2 could provide an explanation for this observation in that when close to or inside Zone 2, RE behavior ranges from non-working to almost working depending on the sign of $\rho$. Also, FE2 and FE4 not using the same finite elements $\left(Q_{2}-Q_{1}\right.$ for FE2 and $Q_{2}-P_{1}^{n c}$ for FE4) could be in different zones.

To conclude this section, although further investigations would be necessary to assess that the proposed explanations are the right ones, we have found that the simple model in equation (9) allowed us to reproduce most of the behavior we have observed in trying to apply RE to the benchmark of this article. 


\section{Conclusions}

In this paper, the methodology that has permitted to establish the first numerical benchmark solutions of a three-dimensional mixed convection flow in a horizontal rectangular channel published in [2] is presented in details. This methodology is based on the use of four different numerical methods (second order FD and FV methods, and third order FE methods), Richardson extrapolations (RE) on very fine grids and cubic spline interpolations.

However the difficulty of the present benchmark problem is that a discontinuity takes place in the thermal gradient over the bottom plate at $x=0$, which not only significantly restricts the conditions of application of $\mathrm{RE}$ to establish reference solutions, but also complicates its analysis. Therefore the theoretical basis of RE is presented and discussed from the viewpoint of this singularity. It is shown that the convergence order, $\alpha$, observed from $\mathrm{RE}$ of the local and integral quantities is reduced to one in the neighborhood of the boundary condition discontinuity and tends to the consistency order, $\alpha^{\circ}=2$ or 3 , of the used discretization methods far from the singularity. It is deduced from this result and other test cases that the problem regularity is close to $r=1$ in the vicinity of the boundary condition discontinuity. Moreover, we have suggested in $\S 4.3$ a modified Taylor expansion to account for the problem singularity in the RE formalism. A simplified model problem has enabled us to reproduce most of the behaviors we have observed in the present benchmark problem and it has helped us to interpret them.

The paper has also brought to the fore several practical difficulties in the proper usage and implementation of RE. It has been shown that the distance between the finest grid solutions and the extrapolated solutions is much smaller for the third order FE2 and FE4 methods than for the second order FD1 and FV3 ones. Furthermore, the local behavior of $\alpha$ is more oscillatory for the two third order methods than for the two second order ones. It was also shown that, for the used grids, RE cannot be applied locally on the whole fields due to the "crossing" of the computed quantities on the different grids. The FE2 and FE4 solutions have appeared very sensitive to these field variations and this behavior has been understood thanks to the model problem introduced in $\S 4.3$. Using larger grid size ratios (resulting in much finer grids) than those required for the present paper would have probably be another way to solve this problem but for much greater computational costs.

It is noteworthy that the four numerical models used for this benchmark have displayed their own sensitivity to the various problem peculiarities (establishment zone, localized thermal gradient singularity, etc.) and, wherever the RE has been found to be applicable, the resulting convergence order could also depend on the quantity (primitive or derived variable) it is based on and its definition ( $L^{2}$ norm, mean value, etc.). This study has also reminded us that the convergence order of a numerical model can be significantly 
deteriorated due to a loss of regularity of the solution and that the standard RE framework should not be used without taking precautions in this case. But, if these precautions are taken, the present study has demonstrated that it is perfectly possible to establish reference solutions with a high accuracy level.

\section{Acknowledgments}

The first author acknowledges Pr. Shihe Xin, from CETHIL, UMR 5008 CNRS/InsaLyon, France, to have provided the finite difference code, FD1, that was developed by him when he was at LIMSI, CNRS, UPR 3251, Orsay, France. The authors acknowledge Donna Calhoun and Sergey Kudriakov for proof reading of the article and providing many useful suggestions. This work was supported by CNRS that provided substantial computational resources on its NEC-SX5 vectorial supercomputer and on its IBM SP4 and SP6 parallel supercomputers at IDRIS, Orsay, France, under project numbers 06-1823 and 07-1823. The third author thanks the Aquitaine Regional Council for the financial support dedicated to a 256-processor cluster investment, located at I2M Institute.

\section{References}

[1] M. Medale, and X. Nicolas, CALL FOR CONTRIBUTIONS: Towards numerical benchmark solutions for 3D mixed convection flows in rectangular channels heated from below, Int. J. Thermal Sc., vol. 45, pp. 331-333, 2006.

[2] X. Nicolas, M. Medale, S. Glockner, and S. Gounand, Benchmark solution for a three-dimensional mixed convection flow - Part 1: reference solutions, Num. Heat Trans. B, vol. 60, issue 5, pp. 325-345, 2011.

[3] H. Pabiou, S. Mergui, and C. Bénard, Wavy secondary instability of longitudinal rolls in Rayleigh-Bénard-Poiseuille flows, J. Fluid Mech., vol. 542, pp. 175-194, 2005 .

[4] W. G. Strang, and G. J. Fix, An analysis of the Finite Element Method, 2nd ed., chap. 8, in Wellesley-Cambridge Press, Cambridge, 1988.

[5] C.H. Marchi, and R. Suero, The lid-driven square cavity flow: Numerical solution with a 1024 x 1024 grid, Journal of the Brazilian Society of Mechanical Sciences and Engineering, vol. 31, pp. 186-198, 2009.

[6] C.-H. Bruneau, and M. Saad, The 2D lid-driven cavity problem revisited. Computers \& Fluids, vol. 35, pp. 326-348, 2006. 
[7] K. F. Alvin, W. L. Oberkampf, B. M. Rutherford, and K. V. Diegert, Methodology for characterizing modeling and discretization uncertainties in computational simulation, (read §4, pp. 28-37), SAND2000-0515, Sandia National Laboratories, Albuquerque, NM, 2000.

[8] P. J. Roache, Perspective: a method for uniform reporting of grid refinement studies, J. Fluid Engineering, vol. 116, pp. 405-413, 1994.

[9] W. L. Oberkampf, and T. Trucano, Verification and validation in computational fluid dynamics, (read §3.3, pp. 31-42), SAND2002-0529, Sandia National Laboratories, Albuquerque, NM, 2002, also in Progress in Aerospace Sciences, vol. 38, pp. 209$272,2002$.

[10] C. J. Roy, Review of code and solution verification procedures for computational simulation, J. Comput. Physics, vol. 205, pp. 131-156, 2005.

[11] G. Evans, and S. Paolucci, The thermoconvective instability of plane Poiseuille flow heated from below: a proposed benchmark solution for open boundary flows, Int. J. Heat Mass Transfer, vol. 11, pp. 1001-1013, 1990.

[12] P. Le Quéré, C. Weisman, H. Paillère, J. Vierendeels, E. Dick, R. Becker, M. Braack, and J. Locke, Modelling of natural convection flows with large temperature differences: a benchmark problem for low Mach number solvers. Part 1. Reference solutions, ESAIM: M2AN, vol. 39, pp. 609-616, 2005.

[13] A. Benzaoui, X. Nicolas, and S. Xin, Efficient vectorized finite difference method to solve the incompressible Navier-Stokes equations for 3D mixed convection flows in high aspect ratio channels, Num. Heat Trans. B, vol. 48, pp. 277-302, 2005.

[14] O. C. Zienkiewicz, and R.L. Taylor, The Finite Element Method, Fourth Edition, vol. 1, Mac Graw Hill, 1994.

[15] W. Shyy, M. Garbey, A. Appukuttan, and J. Wu, Evaluation of Richardson extrapolation in computational fluid dynamics, Numerical Heat Transfer, Part B, vol. 41, pp. 139-164, 2002.

[16] X. Nicolas, M. Medale, S. Gounand, and S. Glockner, Benchmark solution for a three-dimensional mixed convection flow - Detailed technical report, Available online at http://www.sft.asso.fr/Local/sft/dir/user3775/documents/DocumentsDivers/SFT_Report_benchmark_PRB_2011.pdf, pp. 1-44, 2011. 
[17] P. M. Gresho, R. L. Lee, R. L. Sani, M. K. Maslanik, and B. E. Eaton, The consistent Galerkin FEM for computing derived boundary quantities in thermal and/or fluids problems, Int. J. Numer. Meth. Fluids, vol. 7, pp. 1111-1145, 1987.

[18] T. J. R. Hughes, G. Engel, L. Mazzei, and M. G. Larson, The Continuous Galerkin Method is locally conservative, J. Comput. Physics, vol. 163, pp. 467-488, 2000.

[19] M. Garbey, and W. Shyy, A least square extrapolation method for improving solution accuracy of PDE computations, J. Comput. Physics, vol. 186, pp. 1-23, 2003.

\section{Tables}

\begin{tabular}{|c|c|c|c|c|}
\hline Contributor & $\begin{array}{c}N_{x} \times N_{y} \times N_{z} \\
{[\text { symmetry] }}\end{array}$ & $\Delta t$ & $\begin{array}{c}\text { User time } \\
{[\text { computer type (organism/lab)] }}\end{array}$ & $\begin{array}{l}\text { Consistency } \\
\text { orders } \alpha^{\circ}\end{array}$ \\
\hline $\begin{array}{c}\text { MSME, } \\
\text { FD1 }\end{array}$ & $\begin{array}{c}400 \times 134 \times 40 \\
600 \times 200 \times 60 \\
800 \times 268 \times 80 \\
1200 \times 400 \times 120 \\
{[\text { no] }}\end{array}$ & $\begin{array}{l}0.01 \\
0.01 \\
0.002 \\
0.002\end{array}$ & $\begin{array}{c}36 \text { min on } 1 \text { processor } \\
2 h 20 \text { on } 1 \text { processor } \\
25 h \text { on } 1 \text { processor } \\
100 h \text { on } 1 \text { processor } \\
{[\text { NEC SX } 5 \text { (IDRIS)] }}\end{array}$ & $\begin{array}{l}2 \text { for } \theta, u, v, w \\
2 \text { for } p\end{array}$ \\
\hline $\begin{array}{l}\text { IUSTI, } \\
\text { FE2 }\end{array}$ & $\begin{array}{c}601 \times 121 \times 41 \\
901 \times 181 \times 61 \\
1351 \times 271 \times 91 \\
\text { [yes] }\end{array}$ & $\begin{array}{c}0.01 \\
0.01 \\
0.005\end{array}$ & $\begin{array}{l}19 \text { min on } 60 \text { cores } \\
1 h 40 \text { on } 150 \text { cores } \\
43 h 15 \text { on } 225 \text { cores } \\
\text { [IBM SP6 (IDRIS)] }\end{array}$ & $\begin{array}{l}3 \text { for } \theta, u, v, w \\
\quad 2 \text { for } p\end{array}$ \\
\hline $\begin{array}{c}\text { I2M } \\
\text { Institute, } \\
\text { FV3 }\end{array}$ & $\begin{array}{c}601 \times 161 \times 41 \\
901 \times 241 \times 61 \\
1351 \times 361 \times 91 \\
{[\text { yes] }}\end{array}$ & $\begin{array}{l}0.1 \\
0.1 \\
0.1\end{array}$ & $\begin{array}{c}8 h \text { on } 152 \text { cores } \\
12 h \text { on } 152 \text { cores } \\
56 h \text { on } 152 \text { cores } \\
\text { [ALTIX ICE } 8200 \text { (I2M Inst.)] }\end{array}$ & $\begin{array}{l}2 \text { for } \theta, u, v, w \\
\quad 2 \text { for } p\end{array}$ \\
\hline CEA, FE4 & $\begin{array}{c}601 \times 121 \times 49 \\
751 \times 151 \times 61 \\
801 \times 161 \times 65 \\
1001 \times 201 \times 81 \\
\text { [yes] }\end{array}$ & $\begin{array}{l}0.5 \\
0.5 \\
0.5 \\
0.5\end{array}$ & $\begin{array}{c}200 h \text { on } 8 \text { cores } \\
400 h \text { on } 8 \text { cores } \\
450 h \text { on } 8 \text { cores } \\
1600 h \text { on } 8 \text { cores } \\
\text { [PC } 8 \text { cores (CEA)] }\end{array}$ & $\begin{array}{c}3 \text { for } \theta, u, v, w \\
2 \text { for } p\end{array}$ \\
\hline
\end{tabular}

Table 1: Numerical parameters used by the different contributors 


\begin{tabular}{|l|l|l|l|l|l|}
\hline & FD1 & FE2 & FV3 & FE4 & $\begin{array}{l}\text { References } \\
\mathbf{f}_{\text {ref }} \\
\pm \mathbf{f}_{\text {marg }} \\
f_{\text {prec }}=\frac{f_{\text {marg }}}{f_{\text {ref }}}\end{array}$ \\
\hline $2 E_{c}^{f g}$ & 1.292479 & 1.292452 & 1.292355 & 1.292461 & \\
$2 E_{c}^{e x}$ & 1.292446 & 1.292452 & 1.292455 & $1.292467^{\circ}$ & $\mathbf{1 . 2 9 2 4 5 3}$ \\
$\alpha_{E_{c}}$ & 2.22 & 2.92 & 2.00 & -1.92 & $\pm \mathbf{0 . 0 0 0 0 0 8}$ \\
$d_{E_{c}}$ & $2.55 \times 10^{-5}$ & $2.35 \times 10^{-7}$ & $-7.74 \times 10^{-5}$ & $-5.34 \times 10^{-6} \circ$ & $6.19 \times 10^{-6}$ \\
\hline$\Delta P_{i o}^{f g}$ & 14.41210 & 14.40784 & 14.40235 & 14.40694 & \\
$\Delta P_{i o}^{e x}$ & 14.40647 & 14.40649 & 14.40678 & $14.40658^{\circ}$ & $\mathbf{1 4 . 4 0 6 7 0}$ \\
$\alpha_{\Delta P_{i o}}$ & 2.03 & 1.99 & 2.00 & 0.83 & $\pm \mathbf{0 . 0 0 0 2 4}$ \\
$d_{\Delta P_{i o}}$ & $3.91 \times 10^{-4}$ & $9.36 \times 10^{-5}$ & $-3.08 \times 10^{-4}$ & $2.55 \times 10^{-5} \circ$ & $1.67 \times 10^{-5}$ \\
\hline$T_{m}^{f g}$ & 0.448490 & 0.448625 & 0.448725 & 0.448659 & \\
$T_{m}^{e x}$ & 0.448594 & 0.448604 & 0.448606 & 0.448613 & $\mathbf{0 . 4 4 8 6 0 4}$ \\
$\alpha_{T_{m}}^{e x}$ & 1.19 & 1.18 & 1.02 & 1.18 & $\pm \mathbf{0 . 0 0 0 0 1 0}$ \\
$d_{T_{m}}$ & $-2.32 \times 10^{-4}$ & $4.68 \times 10^{-5}$ & $2.65 \times 10^{-4}$ & $1.04 \times 10^{-4}$ & $2.23 \times 10^{-5}$ \\
\hline
\end{tabular}

Table 2: Left columns: finest grid $\left(f^{f g}\right)$ and extrapolated $\left(f^{e x}\right)$ values of the integral functions $f=2 E_{c}, \Delta P_{i o}$ and $T_{m}$, truncation error leading order, $\alpha_{f}$, from their RE and relative distance, $d_{f}=\frac{f^{f g}-f^{e x}}{\left|f^{f g}\right|}$, between the extrapolated and finest grid values. FE4 column: the symbol ${ }^{\circ}$ indicates an erroneous value due to the extrapolation failure (thus the FE4 finest grid value replaces the FE4 extrapolated value in the reference value determination). Right column: reference solutions with their tolerance margin and the precision of their determination. 


\begin{tabular}{|c|c|c|c|c|c|}
\hline & FD1 & FE2 & FV3 & FE4 & $\begin{array}{l}\text { References } \\
\mathbf{f}_{\text {ref }} \pm \mathbf{f}_{\text {marg }} \\
\mathbf{x}_{\text {ref }} \pm \mathbf{x}_{\text {marg }} \\
f_{\text {prec }}=\frac{f_{\text {marg }}}{f_{\text {ref }}}\end{array}$ \\
\hline$\theta_{1}$ & 0.454843 & 0.454844 & 0.454847 & 0.454845 & $(454845 \pm 2) \times 10^{-6}$ \\
\hline$x_{1}$ & 13.696 & 13.691 & 13.692 & 13.691 & $13.693 \pm 0.003$ \\
\hline$\alpha_{1}$ & 1.85 & 3.46 & 1.92 & 2.73 & \\
\hline$d_{1}$ & $3.3 \times 10^{-5}$ & $2.0 \times 10^{-6}$ & $2.2 \times 10^{-4}$ & $1.4 \times 10^{-6}$ & $4.4 \times 10^{-6}$ \\
\hline$\theta_{2}$ & 0.210061 & 0.210048 & 0.210056 & 0.210048 & $(210055 \pm 7) \times 10^{-6}$ \\
\hline$x_{2}$ & 27.319 & 27.315 & 27.332 & 27.313 & $27.322 \pm 0.010$ \\
\hline$\alpha_{2}$ & 1.90 & 3.11 & 1.90 & 4.42 & \\
\hline$d_{2}$ & $-2.4 \times 10^{-3}$ & $2.2 \times 10^{-6}$ & $7.2 \times 10^{-4}$ & $-2.4 \times 10^{-6}$ & $3.3 \times 10^{-5}$ \\
\hline$\overline{u_{1}}$ & 1.572726 & 1.572725 & 1.572713 & 1.572725 & $(1572720 \pm 7) \times 10^{-6}$ \\
\hline$x_{1}$ & 0.950 & 0.945 & 0.944 & 0.941 & $0.945 \pm 0.005$ \\
\hline$\alpha_{1}$ & 2.00 & 3.47 & 2.05 & $* * *$ & \\
\hline$d_{1}$ & $-1.2 \times 10^{-4}$ & $1.3 \times 10^{-6}$ & $3.2 \times 10^{-5}$ & & $4.5 \times 10^{-6}$ \\
\hline$u_{2}$ & 1.660787 & 1.660795 & 1.660826 & 1.660796 & $(1660806 \pm 20) \times 10^{-6}$ \\
\hline$x_{2}$ & 16.299 & 16.295 & 16.291 & 16.289 & $16.294 \pm 0.005$ \\
\hline$\alpha_{2}$ & 1.98 & 1.14 & 2.05 & $* * *$ & \\
\hline$d_{2}$ & $1.9 \times 10^{-4}$ & $1.2 \times 10^{-7}$ & $-8.2 \times 10^{-5}$ & & $1.2 \times 10^{-5}$ \\
\hline$w_{1}$ & 0.0032591 & 0.0032597 & 0.0032605 & 0.0032594 & $(32598 \pm 7) \times 10^{-7}$ \\
\hline$x_{1}$ & 4.265 & 4.258 & 4.259 & 4.252 & $4.259 \pm 0.007$ \\
\hline$\alpha_{1}$ & 1.99 & 3.04 & 1.99 & $* * *$ & \\
\hline$d_{1}$ & $2.5 \times 10^{-4}$ & $-1.2 \times 10^{-4}$ & $-1.6 \times 10^{-3}$ & & $1.8 \times 10^{-4}$ \\
\hline$\overline{w_{2}}$ & -0.472989 & -0.472991 & -0.473026 & -0.472991 & $(-473007 \pm 19) \times 10^{-6}$ \\
\hline$x_{2}$ & 24.907 & 24.901 & 24.898 & 24.898 & $24.902 \pm 0.005$ \\
\hline$\alpha_{2}$ & 2.05 & 3.51 & 1.72 & 4.06 & \\
\hline$d_{2}$ & $-1.8 \times 10^{-4}$ & $-1.1 \times 10^{-6}$ & $1.5 \times 10^{-3}$ & $3.0 \times 10^{-5}$ & $4.0 \times 10^{-5}$ \\
\hline
\end{tabular}

Table 3: Extrapolated values or finest grid values (indicated by $* * *)$ of $f=(\theta, u, w)$ local extrema along the line $(y, z)=(5,0.5)$ and of their streamwise coordinates, $x$; truncation error leading order, $\alpha$, of RE and relative distance, $d=\frac{f^{f g}-f^{e x}}{\left|f^{f g}\right|}$, between the extrapolated and the finest grid values (when the extrapolated value is obtained). In the References column, the reference value and the margin on the primitive variables and on their coordinates are given, as well as the precision on the primitive variables. 


\begin{tabular}{|c|c|c|c|c|c|}
\hline & FD1 & FE2 & FV3 & FE4 & $\begin{array}{l}\text { References } \\
\mathbf{f}_{\text {ref }} \pm \mathbf{f}_{\text {marg }} \\
\mathbf{y}_{\text {ref }} \pm \mathbf{y}_{\text {marg }} \\
f_{\text {prec }}=\frac{f_{\text {marg }}}{f_{\text {ref }}}\end{array}$ \\
\hline$\theta_{1}$ & 0.24719 & 0.24716 & 0.24714 & 0.24715 & $(24716 \pm 3) \times 10^{-5}$ \\
\hline$y_{1}$ & 1.0361 & 1.0368 & 1.0363 & 1.0365 & $1.0364 \pm 0.0004$ \\
\hline$\alpha_{1}$ & 1.90 & 2.91 & 1.96 & 3.38 & $1.2 \times 10^{-4}$ \\
\hline$\theta_{3}$ & 0.77385 & 0.77384 & 0.77387 & 0.77383 & $(77385 \pm 2) \times 10^{-5}$ \\
\hline$y_{3}$ & 3.9042 & 3.9039 & 3.9042 & 3.9041 & $3.9041 \pm 0.0002$ \\
\hline$\alpha_{3}$ & 1.94 & 2.86 & 2.09 & $* * *$ & $2.6 \times 10^{-5}$ \\
\hline$\overline{u_{1}}$ & 1.06513 & 1.06513 & 1.06499 & 1.06509 & $(106506 \pm 7) \times 10^{-5}$ \\
\hline$y_{1}$ & 1.0087 & 1.0087 & 1.0086 & 1.0086 & $1.0086 \pm 0.0001$ \\
\hline$\alpha_{1}$ & 1.95 & 2.87 & 2.01 & $* * *$ & $6.6 \times 10^{-5}$ \\
\hline$u_{3}$ & 1.74979 & 1.74962 & 1.74987 & 1.74978 & $(174975 \pm 13) \times 10^{-5}$ \\
\hline$y_{3}$ & 4.4425 & 4.4425 & 4.4425 & 4.4425 & $4.4425 \pm 0.0000$ \\
\hline$\alpha_{3}$ & 1.94 & 2.94 & 2.02 & $* * *$ & $7.4 \times 10^{-5}$ \\
\hline$v_{1}$ & 0.035892 & 0.035912 & 0.035916 & 0.035900 & $(35904 \pm 12) \times 10^{-6}$ \\
\hline$y_{1}$ & 0.7049 & 0.7043 & 0.7050 & 0.7051 & $0.7047 \pm 0.0004$ \\
\hline$\alpha_{1}$ & 1.97 & 3.15 & 2.03 & $* * *$ & $3.3 \times 10^{-4}$ \\
\hline$v_{3}$ & 0.032867 & 0.032907 & 0.032917 & 0.032878 & $(32892 \pm 25) \times 10^{-6}$ \\
\hline$y_{3}$ & 4.7390 & 4.7395 & 4.7390 & 4.7388 & $4.7391 \pm 0.0004$ \\
\hline$\alpha_{3}$ & 1.96 & $* * *$ & 1.67 & 3.62 & $7.6 \times 10^{-4}$ \\
\hline$\overline{w_{1}}$ & 0.372397 & 0.372372 & 0.372496 & 0.372397 & $(37243 \pm 6) \times 10^{-5}$ \\
\hline$y_{1}$ & 0.2286 & 0.2285 & 0.2285 & 0.2285 & $0.2285 \pm 0.0001$ \\
\hline$\alpha_{1}$ & 2.00 & 2.91 & 1.98 & $* * *$ & $1.6 \times 10^{-4}$ \\
\hline$\overline{w_{3}}$ & 0.490347 & 0.490335 & 0.490478 & 0.490347 & $(49041 \pm 7) \times 10^{-5}$ \\
\hline$y_{3}$ & 3.9028 & 3.9029 & 3.9027 & 3.9028 & $3.9028 \pm 0.0001$ \\
\hline$\alpha_{3}$ & 1.95 & 2.91 & 1.96 & $* * *$ & $1.4 \times 10^{-4}$ \\
\hline
\end{tabular}

Table 4: Extrapolated values or finest grid values (indicated by $* * *)$ of $f=(\theta, u, v, w)$ local extrema along the line $(x, z)=(30,0.5)$ and of their spanwise coordinates, $y$, and truncation error leading order, $\alpha$, of RE. See the Table 3 caption for the description of the References column. Other extrema values at intermediate coordinates $y_{2}$ are given in [16]. 


\begin{tabular}{|c|c|c|c|c|c|}
\hline & FD1 & FE2 & FV3 & FE4 & $\begin{array}{l}\text { References } \\
\mathbf{f}_{\text {ref }} \pm \mathbf{f}_{\text {marg }} \\
\mathbf{x}_{\text {ref }} \pm \mathbf{x}_{\text {marg }} \\
f_{\text {prec }}=\frac{f_{\text {marg }}}{f_{\text {ref }}}\end{array}$ \\
\hline $\begin{array}{l}N u_{t 1} \\
x_{1} \\
\alpha_{1} \\
d_{1} \\
N u_{t 1}^{\text {cons }} \\
x_{1}^{\text {cons }}\end{array}$ & $\begin{array}{l}0.44151 \\
21.107 \\
1.86 \\
1.3 \times 10^{-3}\end{array}$ & $\begin{array}{l}0.44144 \\
21.106 \\
1.68 \\
9.5 \times 10^{-4}\end{array}$ & $\begin{array}{l}0.44119 \\
21.110 \\
1.95 \\
3.1 \times 10^{-3}\end{array}$ & $\begin{array}{l}0.44145 \\
21.105 \\
1.68 \\
1.1 \times 10^{-3} \\
0.44150 \\
21.101\end{array}$ & $\begin{array}{l}0.44135 \pm 0.00016 \\
21.106 \pm 0.005 \\
3.6 \times 10^{-4}\end{array}$ \\
\hline $\begin{array}{l}N u_{t 2} \\
x_{2} \\
\alpha_{2} \\
d_{2} \\
N u_{t 2}^{\text {cons }} \\
x_{2}^{\text {cons }} \\
\end{array}$ & $\begin{array}{l}0.60675 \\
28.085 \\
1.90 \\
-2.5 \times 10^{-3}\end{array}$ & $\begin{array}{l}0.60658 \\
28.085 \\
1.70 \\
1.2 \times 10^{-3}\end{array}$ & $\begin{array}{l}0.60615 \\
28.074 \\
1.90 \\
2.9 \times 10^{-3}\end{array}$ & $\begin{array}{l}0.60657 \\
28.081 \\
1.68 \\
1.4 \times 10^{-3} \\
0.60666 \\
28.077 \\
\end{array}$ & $\begin{array}{l}\mathbf{0 . 6 0 6 4 5} \pm \mathbf{0 . 0 0 0 3 0} \\
\mathbf{2 8 . 0 8 0} \pm \mathbf{0 . 0 0 6} \\
4.9 \times 10^{-4}\end{array}$ \\
\hline $\begin{array}{l}N u_{b 1} \\
x_{1} \\
\alpha_{1} \\
d_{1} \\
N u_{b 1}^{\text {cons }} \\
x_{1}^{\text {cons }} \\
\end{array}$ & $\begin{array}{l}3.48657 \\
24.997 \\
2.00 \\
1.6 \times 10^{-3}\end{array}$ & $\begin{array}{l}3.48650 \\
24.990 \\
3.05 \\
2.0 \times 10^{-4}\end{array}$ & $\begin{array}{l}3.4416^{\circ} \\
25.037^{\circ} \\
* * *\end{array}$ & $\begin{array}{l}3.48650 \\
24.990 \\
3.11 \\
2.8 \times 10^{-4} \\
3.48663 \\
24.988\end{array}$ & $\begin{array}{l}\mathbf{3 . 4 8 6 5 7} \pm \mathbf{0 . 0 0 0 0 7} \\
\mathbf{2 4 . 9 9 3} \pm \mathbf{0 . 0 0 5} \\
2.0 \times 10^{-5}\end{array}$ \\
\hline $\begin{array}{l}N u_{b 2} \\
x_{2} \\
\alpha_{2} \\
d_{2} \\
N u_{b 2}^{\text {cons }} \\
x_{2}^{\text {cons }} \\
\end{array}$ & $\begin{array}{l}3.38972 \\
29.165 \\
2.06 \\
1.8 \times 10^{-3}\end{array}$ & $\begin{array}{l}3.38945 \\
29.165 \\
3.06 \\
1.9 \times 10^{-4}\end{array}$ & $\begin{array}{l}3.3455^{\circ} \\
29.222^{\circ} \\
* * *\end{array}$ & $\begin{array}{l}3.38945 \\
29.164 \\
3.11 \\
2.7 \times 10^{-4} \\
3.38958 \\
29.162 \\
\end{array}$ & $\begin{array}{l}3.38959 \pm 0.00014 \\
29.164 \pm \mathbf{0 . 0 0 2} \\
4.1 \times 10^{-5}\end{array}$ \\
\hline
\end{tabular}

Table 5: Extrapolated values or finest grid values (indicated by $* * *)$ of $f=\left(N u_{t}, N u_{b}\right)$ local extrema along the line $y=5$ and of their streamwise coordinates, $x$; truncation error leading order, $\alpha$, of RE and relative distance, $d=\frac{f^{f g}-f^{e x}}{\left|f^{f g}\right|}$, between the extrapolated and finest grid values (when the extrapolated value is got). In the reference column, the reference value and the margin on the Nusselt numbers and on their coordinates are given, as well as the precision on the Nusselt numbers. For FE4 contribution, $N u_{t}$ and $N u_{b}$ are the extrapolated values of the non consistent Nusselt numbers and $N u_{t}^{\text {cons }}$ and $N u_{b}^{\text {cons }}$ are the consistent Nusselt number values on the finest grid. The symbol ${ }^{\circ}$ indicates values that are excluded from the reference determination. 


\section{Figure captions}

Figure 1: Geometry and thermal boundary conditions on the top and bottom walls (the vertical lateral walls are adiabatic). The red dotted lines are the lines along which $\theta, u, v$ and $w$ profiles and their extrema are calculated.

Figure 2: On the left, the square domain (1) with a crack is used by Strang and Fix [4] to study the effect of the singularity at point B. On the right, the equivalent domain

(2) is obtained using the symmetry of domain (1) through the line ABC. The domain (2) has the same boundary condition singularity at point B as the present benchmark problem for the temperature at $x=0$ and $z=0$ or 1 (see Figure 1 ).

Figure 3: FD1 solutions of the vertical velocity component streamwise profiles along the line at $(y, z)=(B / 5,0.5)$, computed on the four grids described in Table 1 , together with the extrapolated solution. The latter can be considered as the asymptotic solution here because the $\alpha$ values monotonously vary between 2.01 and 2.16 when $x$ varies between 24.7 and 25.3. In this figure, all the symbols correspond to the computational or extrapolation points and the curves to the cubic spline interpolation curves. The small window zooms in the local extremum of the extrapolated curve. The value $w_{2}=-0.472989$ and the coordinate $x_{2}=24.907$ of this extremum are reported in Table 3.

Figure 4: Space evolution of the convergence orders $\alpha$ observed from RE of the integral quantities $T_{m}, T_{L^{2}}$ and $W_{L^{2}}$ in various upstream or downstream subdomains of the computational domain.

Figure 5: FD1 solutions of the temperature streamwise profiles along the line at $(y, z)=(B / 5,0.5)$, computed on the four grids described in Table 1 , together with the extrapolated solution. The latter does not tend to the asymptotic solution near $x=22.35$ because the $\alpha$ values (the black filled circles in the figure) diverge where the profiles intersect. All the symbols correspond to the computational or extrapolated points. The curves correspond to the cubic spline interpolation curves, except for the extrapolated solution where they are linear segments linking the extrapolated points.

Figure 6: Comparison of the streamwise profiles of the extrapolation coefficients, $\alpha$, 
computed by the four contributors for $\theta, u$ and $w$, along the line at $(y, z)=(2,0.2)$. The corresponding profiles of $\theta_{f g}, u_{f g}$ and $w_{f g}$ on the finest grid are also drawn on the figures. Similar profiles for $v$ are available in [16].

Figure 7: Comparison of streamwise and spanwise profiles of the extrapolation coefficients, $\alpha$, for $N u_{t}$ computed by the four contributors. The corresponding profiles of $N u_{t-f g}$ on the finest grid are also drawn on the figures.

Figure 8: Profiles of $\alpha$ and $\sigma$ as a function of $\log |\rho|$. 


\section{Figures}

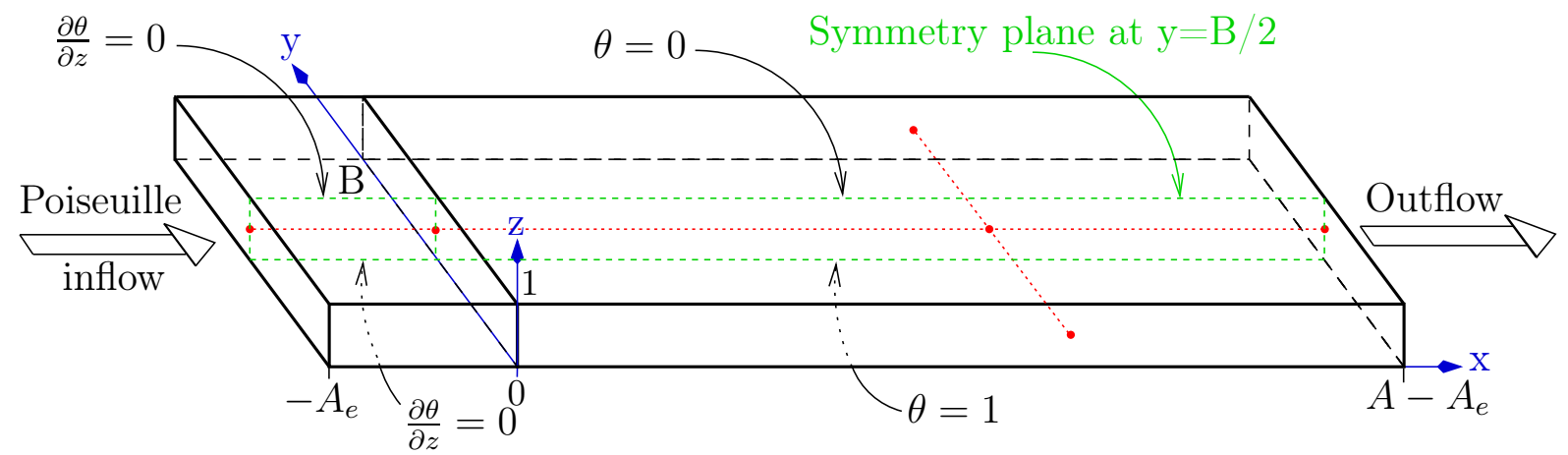

Figure 1: Geometry and thermal boundary conditions on the top and bottom walls (the vertical lateral walls are adiabatic). The red dotted lines are the lines along which $\theta, u, v$ and $w$ profiles and their extrema are calculated. 


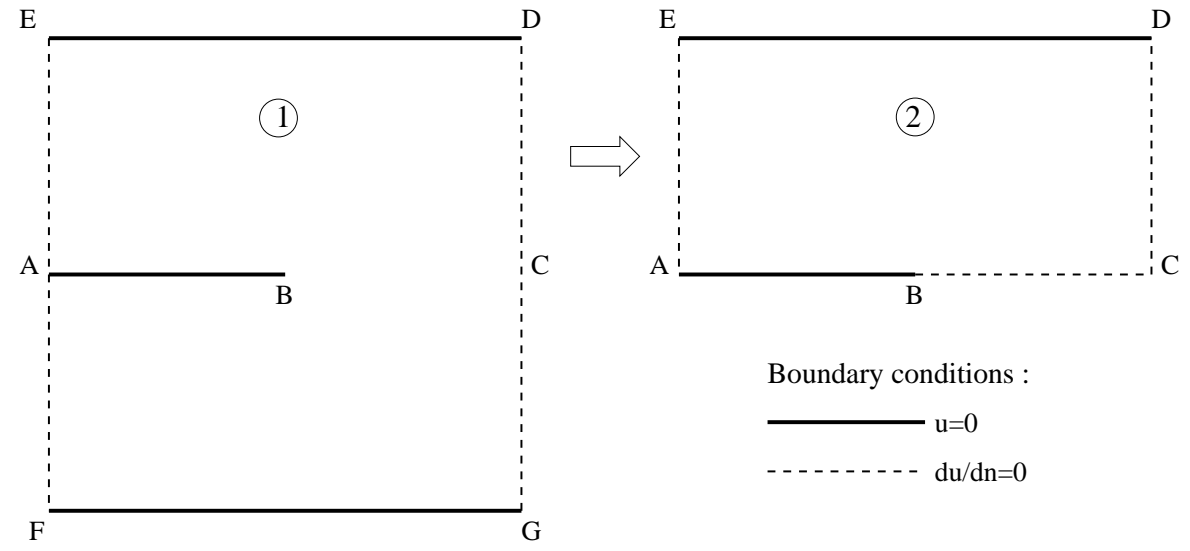

Figure 2: On the left, the square domain (1) with a crack is used by Strang and Fix [4] to study the effect of the singularity at point B. On the right, the equivalent domain (2) is obtained using the symmetry of domain (1) through the line ABC. The domain (2) has the same boundary condition singularity at point B as the present benchmark problem for the temperature at $x=0$ and $z=0$ or 1 (see Figure 1 ). 


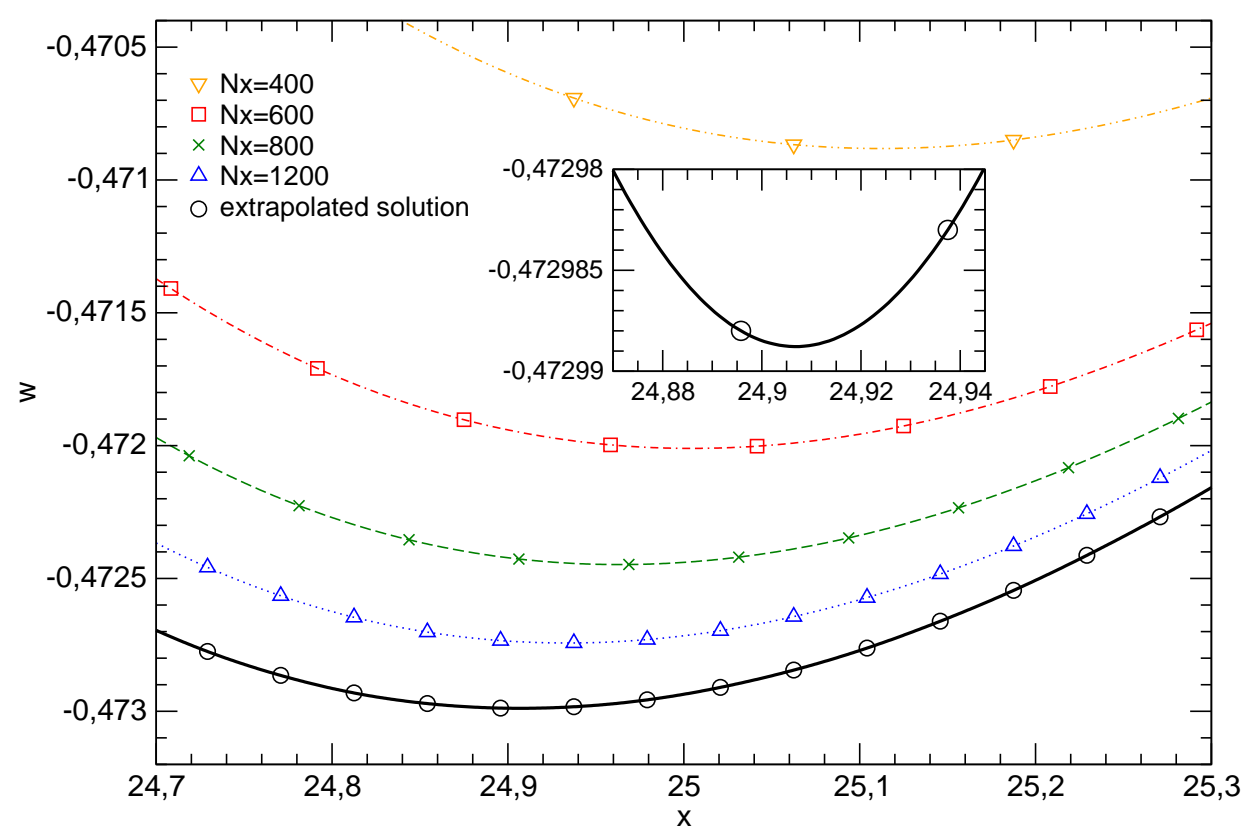

Figure 3: FD1 solutions of the vertical velocity component streamwise profiles along the line at $(y, z)=(B / 5,0.5)$, computed on the four grids described in Table 1 , together with the extrapolated solution. The latter can be considered as the asymptotic solution here because the $\alpha$ values monotonously varies between 2.01 and 2.16 when $x$ varies between 24.7 and 25.3. In this figure, all the symbols correspond to the computational or extrapolation points and the curves to the cubic spline interpolation curves. The small window zooms in the local extremum of the extrapolated curve. The value $w_{2}=$ -0.472989 and the coordinate $x_{2}=24.907$ of this extremum are reported in Table 3 . 


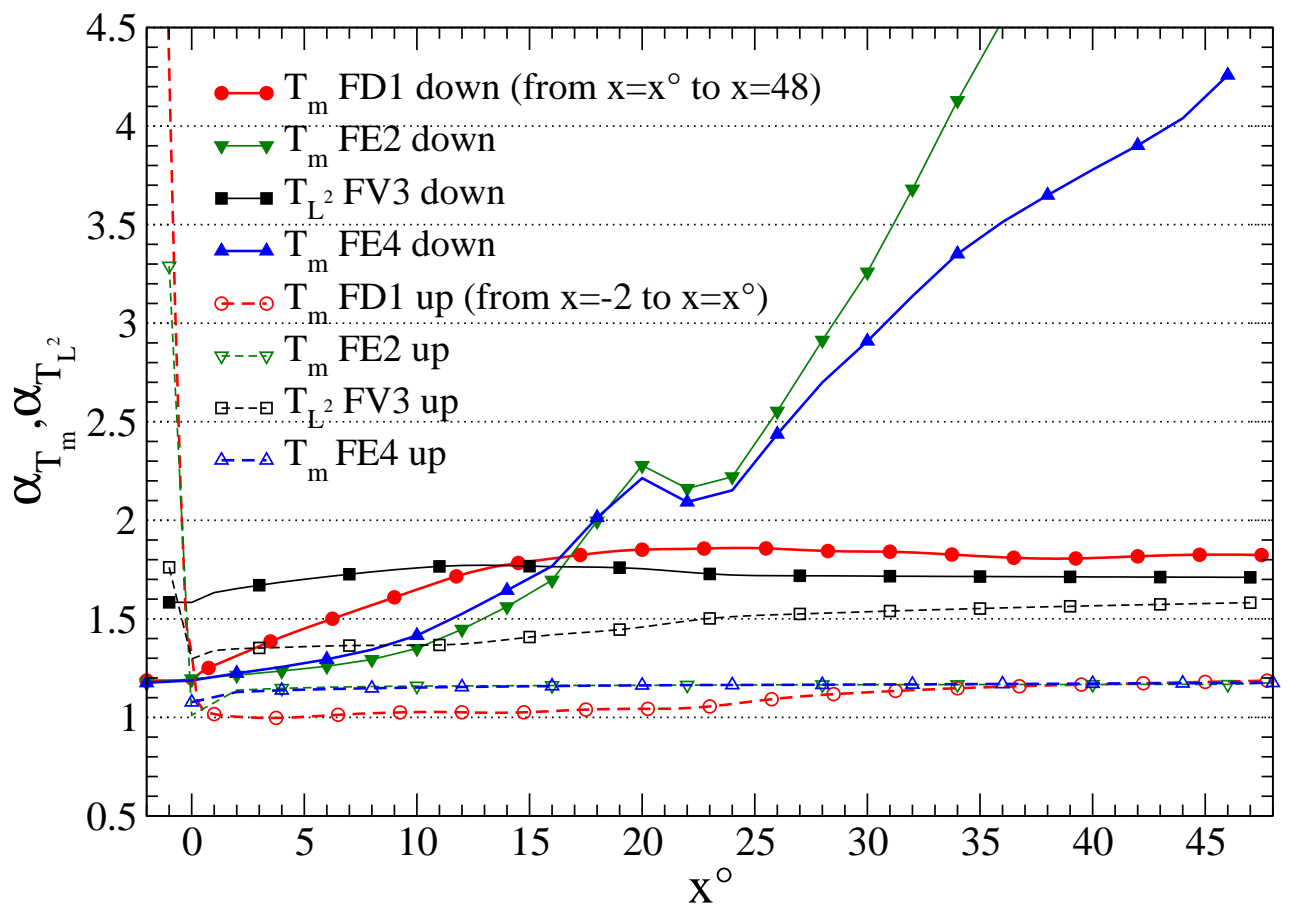

(a) Average temperature $T_{m}$ or $L^{2}$ norm of temperature $T_{L^{2}}$

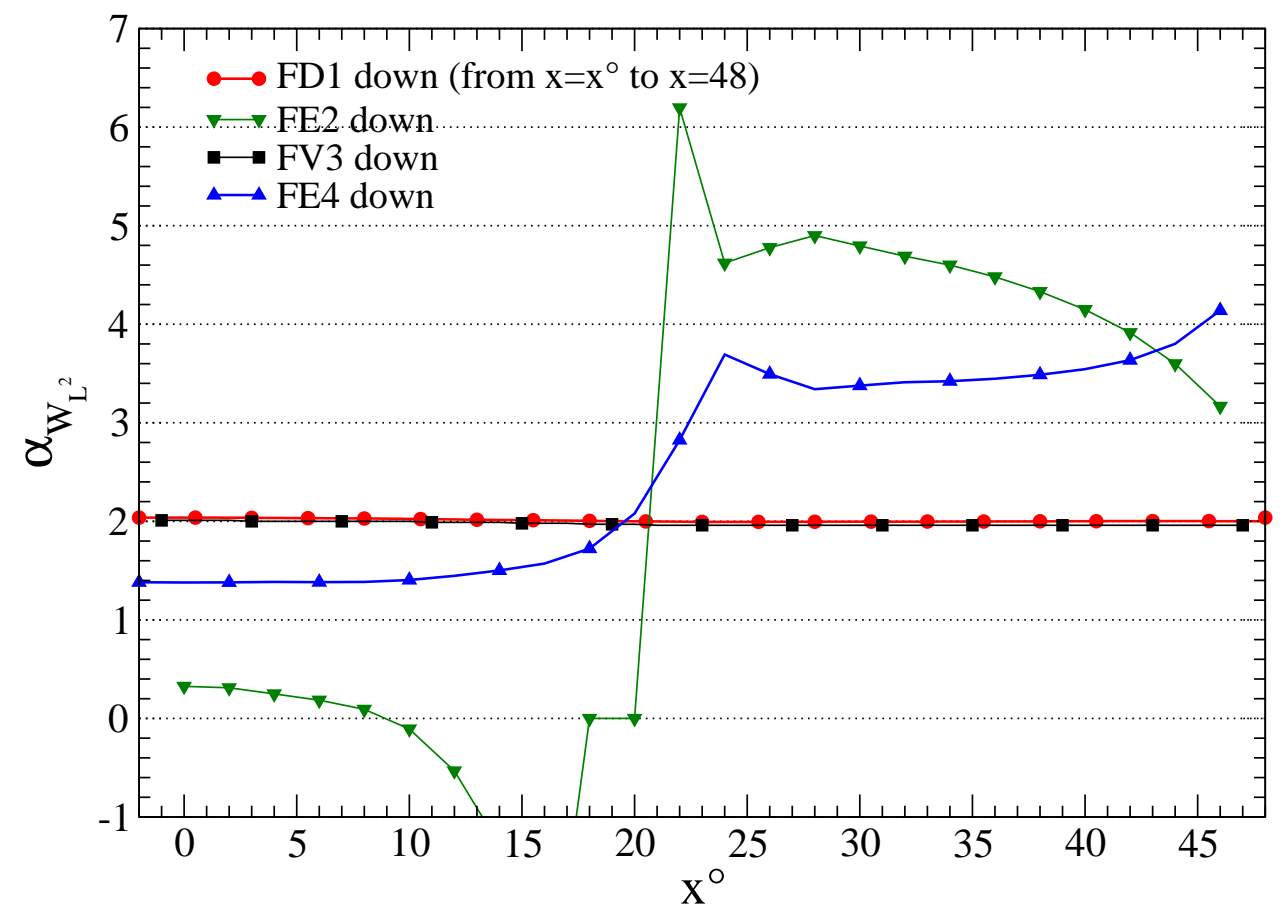

(b) $L^{2}$ norm of vertical velocity component $W_{L^{2}}$

Figure 4: Space evolution of the convergence orders $\alpha$ observed from RE of the integral quantities $T_{m}, T_{L^{2}}$ and $W_{L^{2}}$ in various upstream or downstream subdomains of the computational domain. 


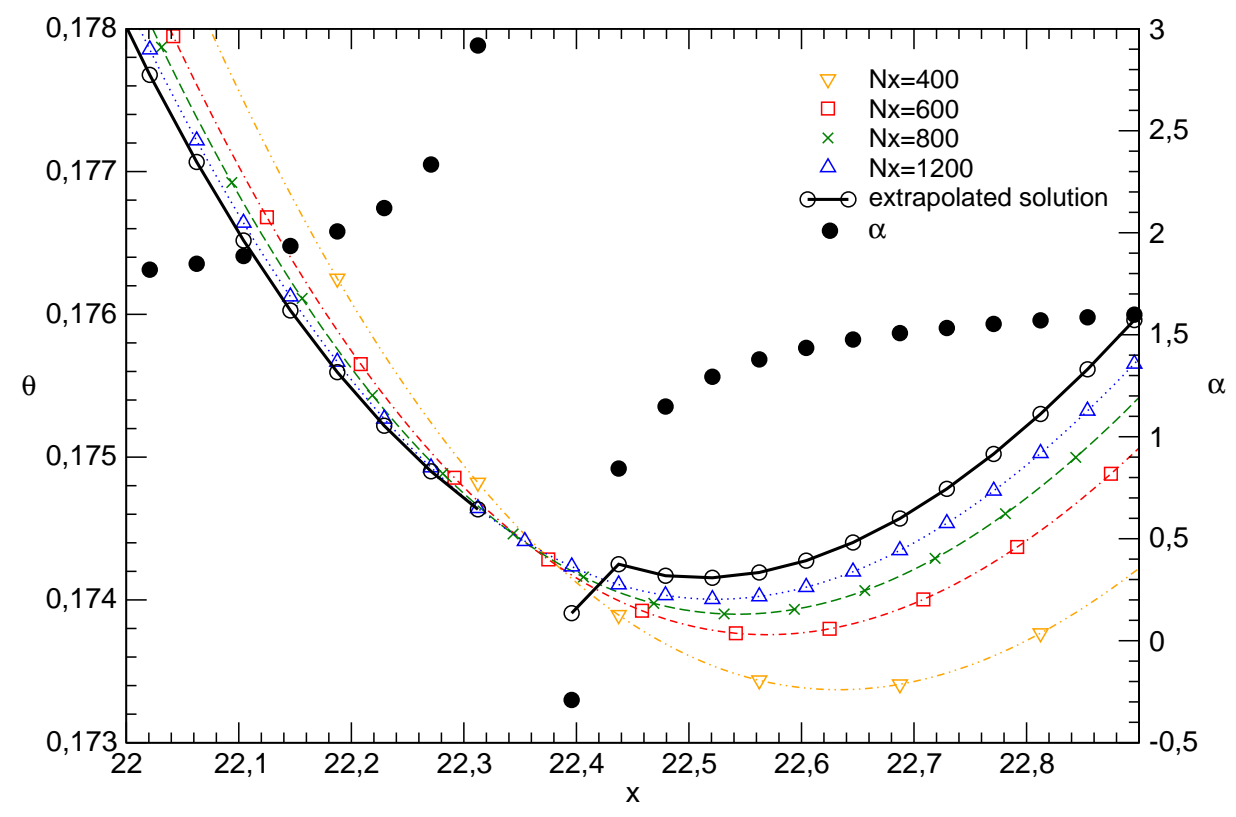

Figure 5: FD1 solutions of the temperature streamwise profiles along the line at $(y, z)=(B / 5,0.5)$, computed on the four grids described in Table 1, together with the extrapolated solution. The latter does not tend to the asymptotic solution near $x=22.35$ because the $\alpha$ values (the black filled circles in the figure) diverge where the profiles intersect. All the symbols correspond to the computational or extrapolated points. The curves correspond to the cubic spline interpolation curves, except for the extrapolated solution where they are linear segments linking the extrapolated points. 

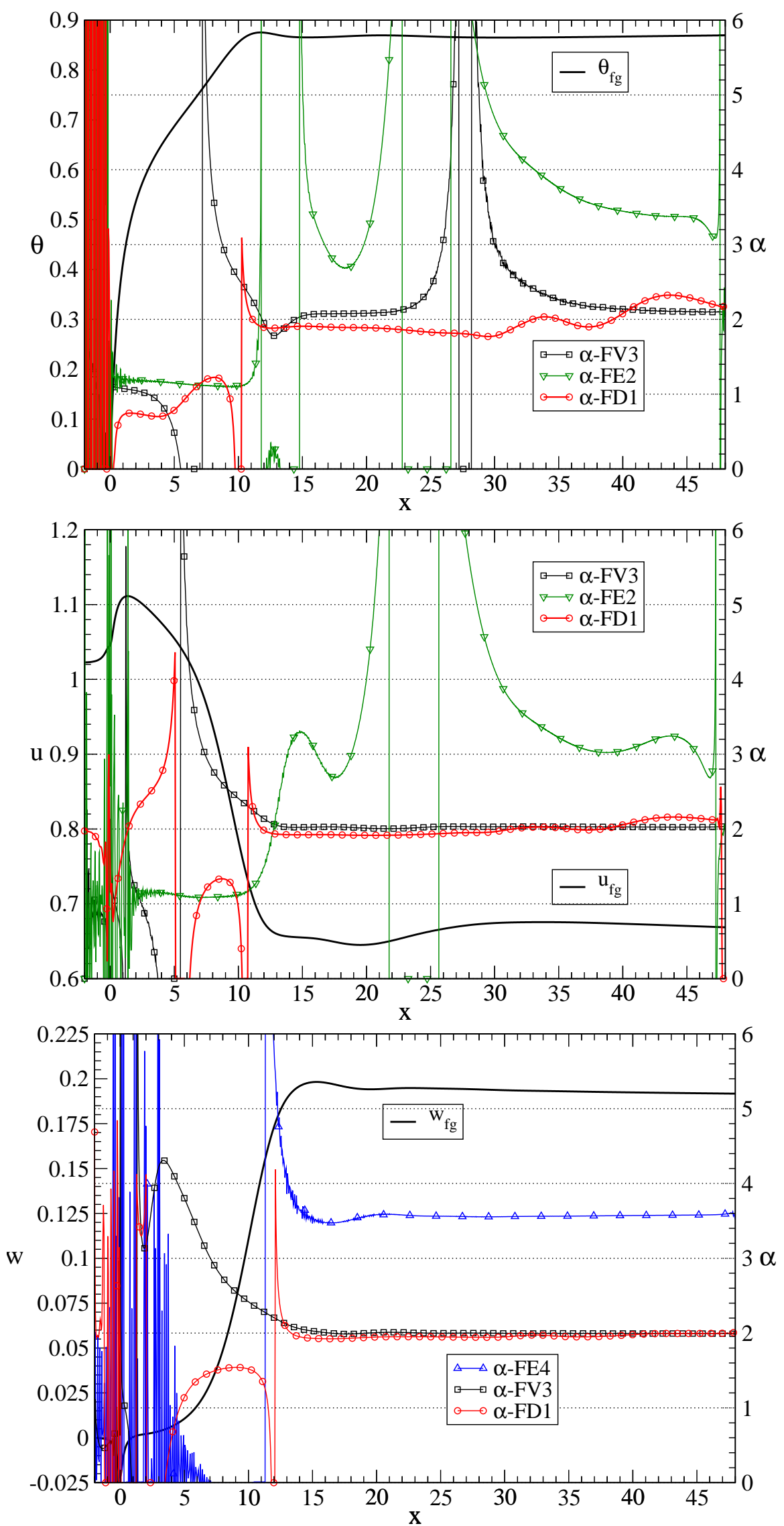

Figure 6: Comparison of the streamwise profiles of the extrapolation coefficients, $\alpha$, computed by the four contributors for $\theta, u$ and $w$, along the line at $(y, z)=(2,0.2)$. The corresponding profiles of $\theta_{f g}, u_{f g}$ and $w_{f g}$ on the finest grid are also drawn on the figures. Similar profiles for $v$ are available in [16]. 33 


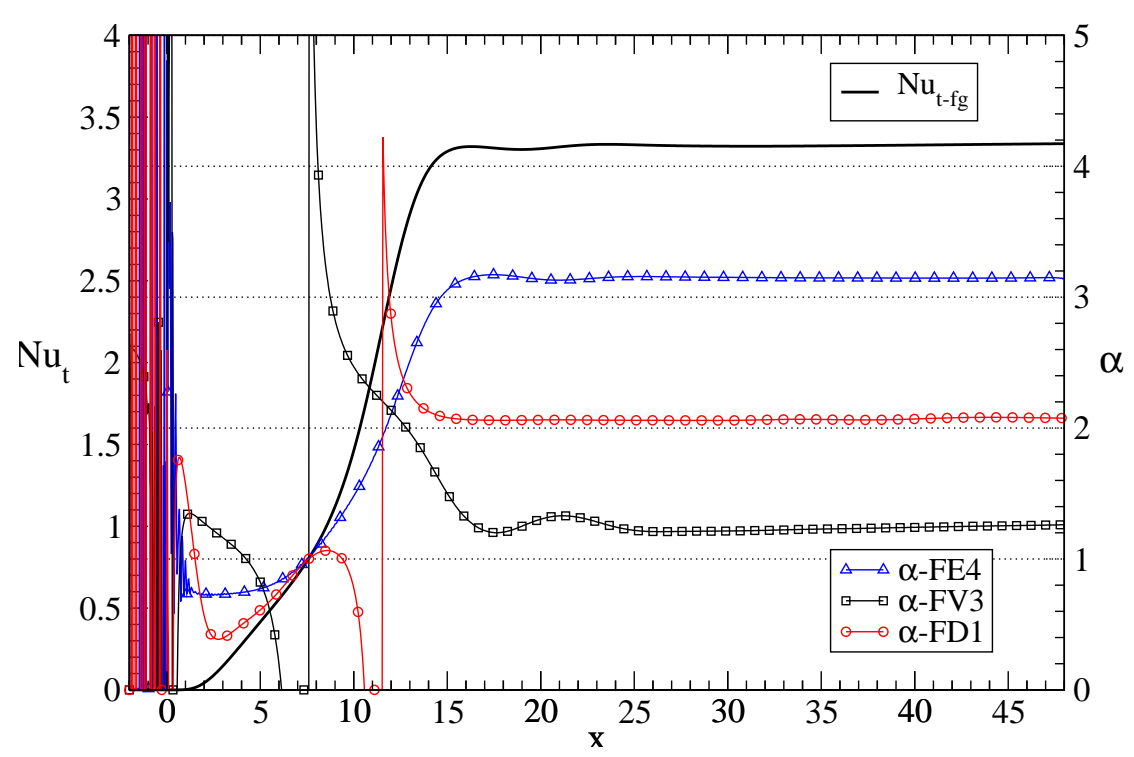

(a) $(y, z)=(2,1)$

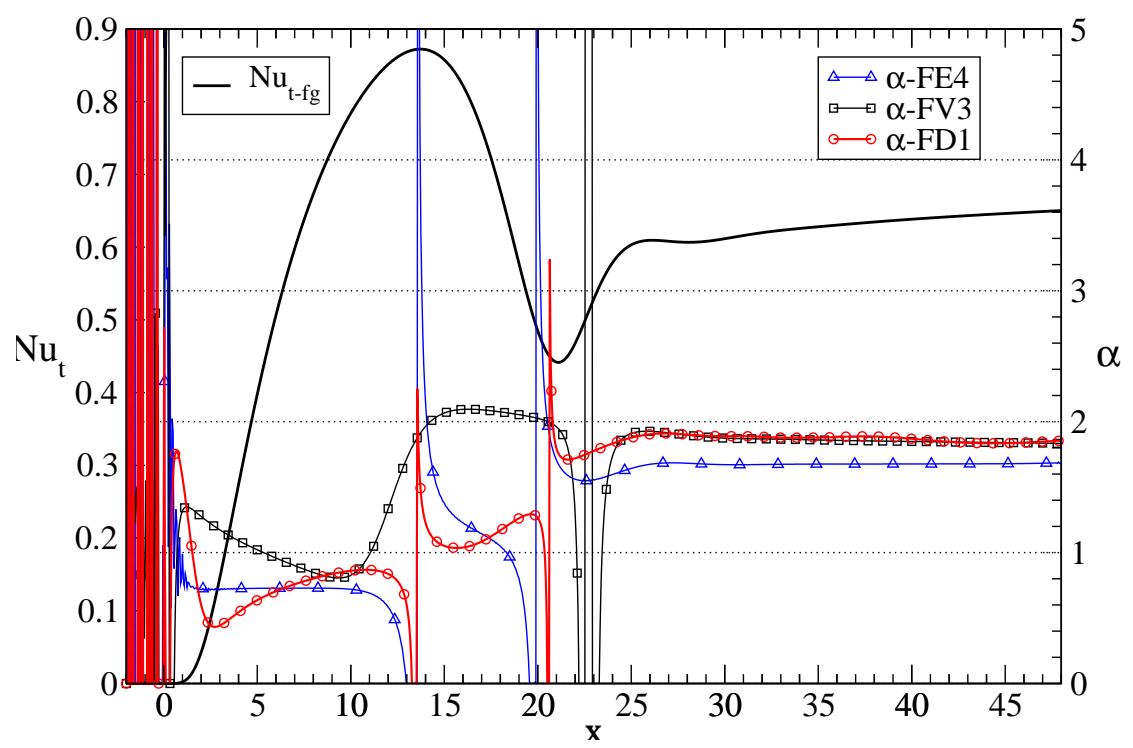

(b) $(y, z)=(5,1)$

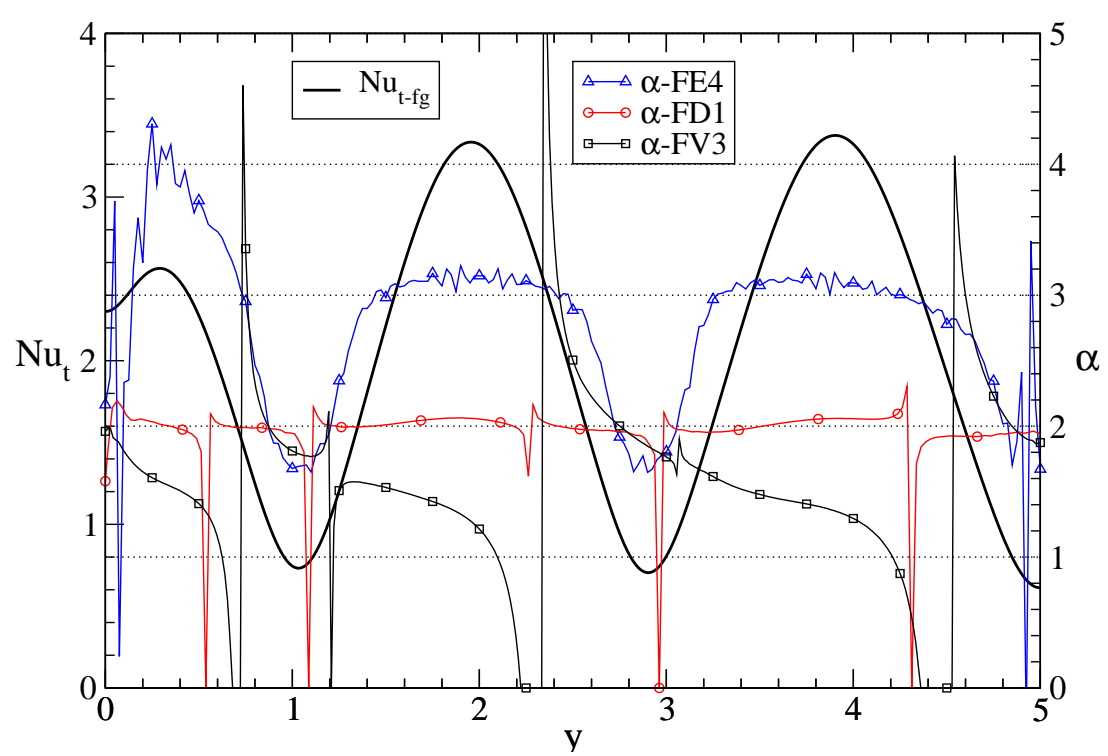

(c) $(x, z)=(30,1)$

Figure 7: Comparison of streamwise and spanwise profiles of the extrapolation coefficients, $\alpha$, for $N u_{t}$ computed by the four contributors. The corresponding profiles of $N u_{t-f g}$ on the finest grid are also drawn on the figures. 


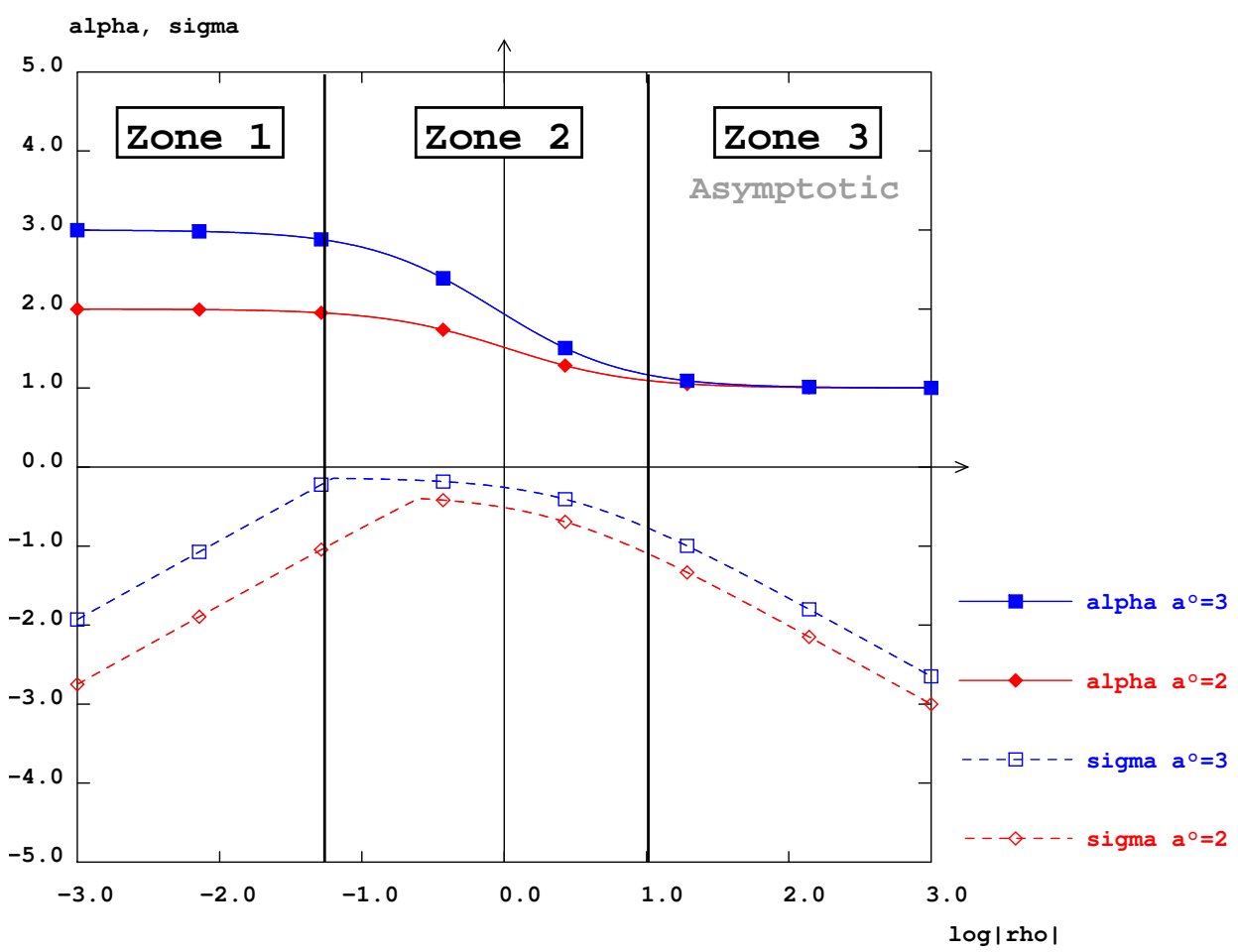

(a) $\alpha^{\circ}=2$ or 3 and $\rho>0$.

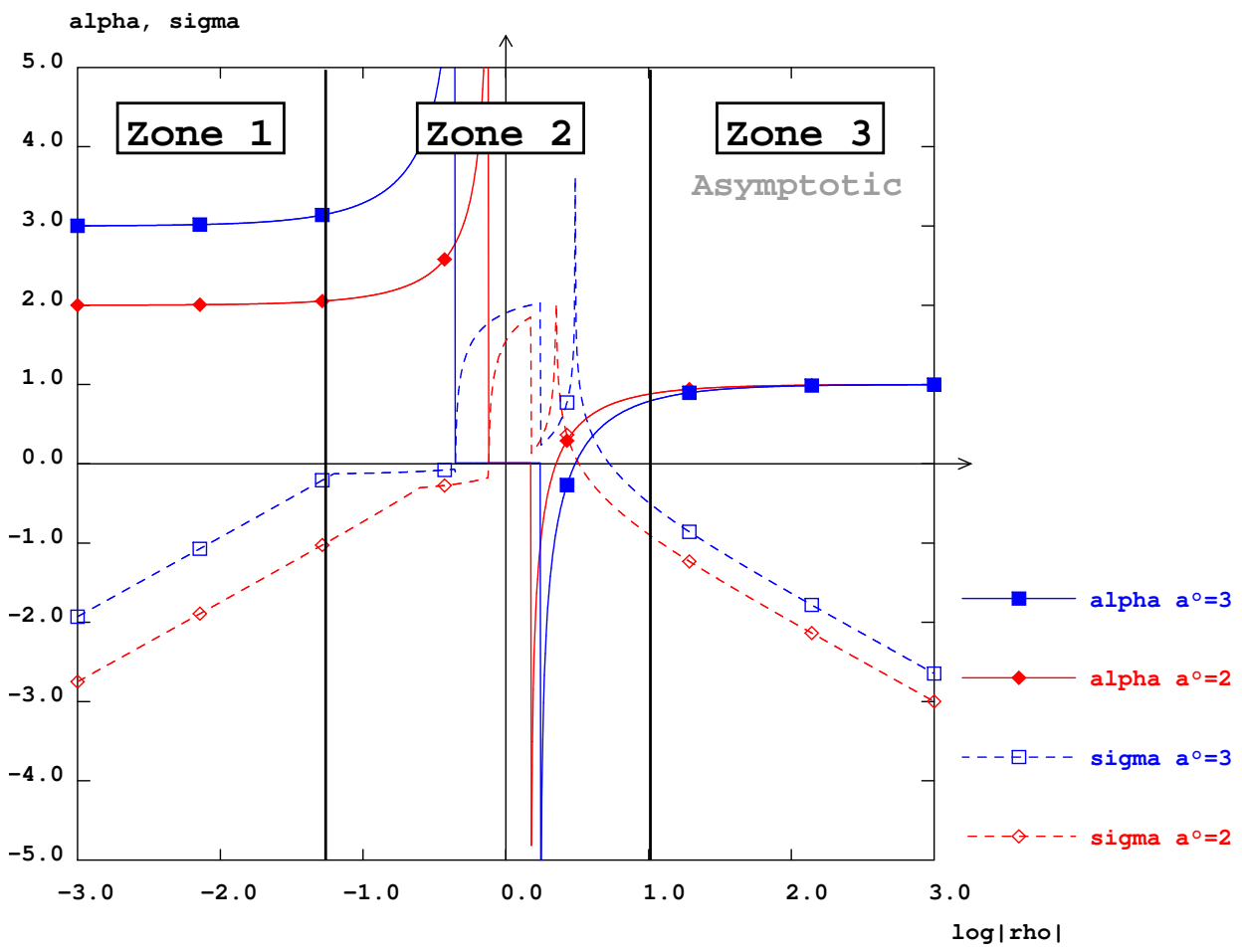

(b) $\alpha^{\circ}=2$ or 3 and $\rho<0$.

Figure 8: Profiles of $\alpha$ and $\sigma$ as a function of $\log |\rho|$. 